\title{
Methylation in pericytes after acute injury promotes chronic kidney disease
}

\author{
Yu-Hsiang Chou, ${ }^{1,2,3}$ Szu-Yu Pan, ${ }^{1,3,4}$ Yu-Han Shao, ${ }^{3}$ Hong-Mou Shih, ${ }^{3,5}$ Shi-Yao Wei, ${ }^{3,6}$ Chun-Fu Lai, ${ }^{1}$ Wen-Chih Chiang, ${ }^{1}$ \\ Claudia Schrimpf, ${ }^{7}$ Kai-Chien Yang, ${ }^{8,9}$ Liang-Chuan Lai, ${ }^{3}$ Yung-Ming Chen, ${ }^{1}$ Tzong-Shinn Chu, ${ }^{1}$ and Shuei-Liong Lin ${ }^{1,3,10,11}$
}

\begin{abstract}
'Renal Division, Department of Internal Medicine, National Taiwan University Hospital, Taipei, Taiwan. ${ }^{2}$ Department of Internal Medicine, National Taiwan University Hospital Jin-Shan Branch, New Taipei City, Taiwan. ${ }^{3}$ Graduate Institute of Physiology, College of Medicine, National Taiwan University, Taipei, Taiwan. ${ }^{4}$ Renal Division, Department of Internal Medicine, Far Eastern Memorial Hospital, New Taipei City, Taiwan. ${ }^{5}$ Division of Nephrology, Department of Internal Medicine, MacKay Memorial Hospital, Taipei, Taiwan. ${ }^{6}$ Department of Nephrology, Second Affiliated Hospital of Harbin Medical University, Harbin, China. 'Department of Cardiothoracic, Transplantation and Vascular Surgery, Hannover Medical School, Hannover, Cermany. ${ }^{8}$ Craduate Institute of Pharmacology, College of Medicine, National Taiwan University, Taipei, Taiwan. ${ }^{~}$ Division of Cardiology, Department of Internal Medicine, and ${ }^{10}$ Department of Integrated Diagnostics and Therapeutics, National Taiwan University Hospital, Taipei, Taiwan. "Research Center for Developmental Biology and Regenerative Medicine, National Taiwan University, Taipei, Taiwan.
\end{abstract}

\begin{abstract}
The origin and fate of renal myofibroblasts is not clear after acute kidney injury (AKI). Here, we demonstrate that myofibroblasts were activated from quiescent pericytes (qPericytes) and the cell numbers increased after ischemia/ reperfusion injury-induced AKI (IRI-AKI). Myofibroblasts underwent apoptosis during renal recovery but one-fifth of them survived in the recovered kidneys on day 28 after IRI-AKI and their cell numbers increased again after day 56. Microarray data showed the distinctive gene expression patterns of qPericytes, activated pericytes (aPericytes, myofibroblasts), and inactivated pericytes (iPericytes) isolated from kidneys before, on day 7, and on day 28 after IRI-AKI. Hypermethylation of the Acta2 repressor $Y b \times 2$ during IRI-AKI resulted in epigenetic modification of iPericytes to promote the transition to chronic kidney disease (CKD) and aggravated fibrogenesis induced by a second AKI induced by adenine. Mechanistically, transforming growth factor- $\beta 1$ decreased the binding of YBX2 to the promoter of Acta2 and induced $Y b \times 2$ hypermethylation, thereby increasing $\alpha$-smooth muscle actin expression in aPericytes. Demethylation by 5 -azacytidine recovered the microvascular stabilizing function of aPericytes, reversed the profibrotic property of iPericytes, prevented AKI-CKD transition, and attenuated fibrogenesis induced by a second adenine-AKI. In conclusion, intervention to erase hypermethylation of pericytes after AKI provides a strategy to stop the transition to CKD.
\end{abstract}

\section{Introduction}

The conventional wisdom that survivors with full functional recovery from acute kidney injury (AKI) tend to have a good prognosis appears to be flawed $(1,2)$. Ongoing renal pathophysiological processes after AKI may continue for a duration between 7 and 90 days or beyond, disease courses defined as acute kidney disease (AKD) and chronic kidney disease (CKD), respectively $(3,4)$. That is, AKI, AKD, and CKD form a continuum whereby the initial kidney injury leads to ongoing renal injury and eventually end-stage renal disease if no effective treatment is provided (1-3). The known pathogenic mechanisms of the AKI-CKD transition include maladaptive repair, cell cycle (G2/M) arrest of tubular epithelial cells, perpetuated fibroblast activation, microvascular rarefaction, chronic inflammation, and sustained renin-angiotensin system (RAS) activation after AKI (4-12). At present, the only potentially effective treatment to prevent CKD has been RAS blockade during AKD $(4,13,14)$, suggesting a huge unmet medical need.

Conflict of interest: The authors have declared that no conflict of interest exists. Copyright: () 2020, American Society for Clinical Investigation.

Submitted: December 16, 2019; Accepted: May 21, 2020; Published: August 4, 2020

Reference information: J Clin Invest. 2020;130(9):4845-4857.

https://doi.org/10.1172/JCl135773.
Kidney pericytes are cells of mesenchymal origin that express platelet-derived growth factor receptor- $\beta$ (PDGFR $\beta^{+}$) and collagen. Pericytes attach to capillaries that share a common basement membrane and form junctions with endothelial cells to support microvasculature, regulate blood flow, and produce erythropoietin (EPO) $(9,15-21)$. Previous studies have shown that pericytes are the progenitor cells of scar-producing myofibroblasts in CKD with progressive fibrosis $(15,22-24)$. Myofibroblasts express a high level of $\alpha$-smooth muscle actin ( $\alpha \mathrm{SMA}$ ) and migrate away from endothelial cells, actively proliferate and produce excessive amounts of extracellular matrix (ECM), thereby leading to scar formation and kidney atrophy. Meanwhile, myofibroblasts lose the function of microvascular stabilization and EPO production $(9,17,25)$. In AKI with functional recovery, however, the fate and role of pericytes are poorly understood.

Selective pericyte ablation leads to AKI with endothelial cell damage, capillary rarefaction, and tubular injury $(18,19)$. In the absence of pericytes, however, renal fibrosis is mild despite the occurrence of AKI, echoing the crucial role of pericytes as the progenitor cells of myofibroblasts in CKD. In contrast, selective tubular cell ablation causes transient activation, proliferation, and migration of pericytes to enclose the injured tubular epithelium and promote tubular regeneration (26). In line with these findings, our previous study demonstrated the transient increase 
A

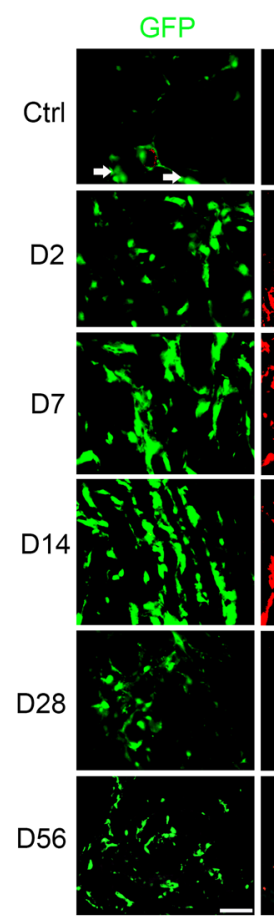

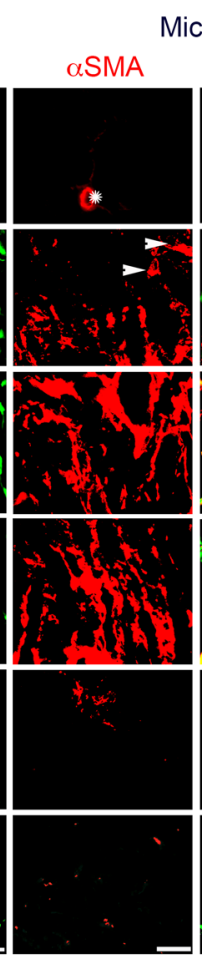

Mice:Col1a1-GFPTg

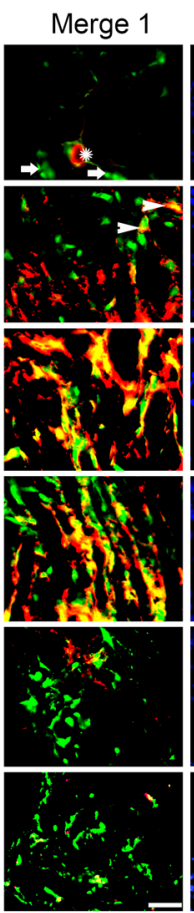

\section{DAP}

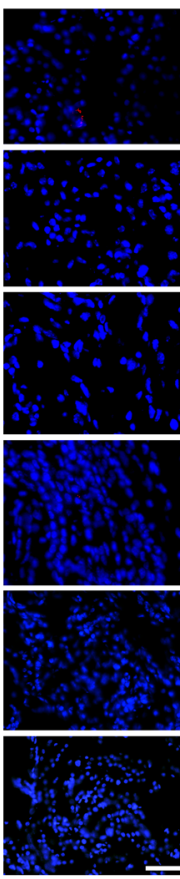

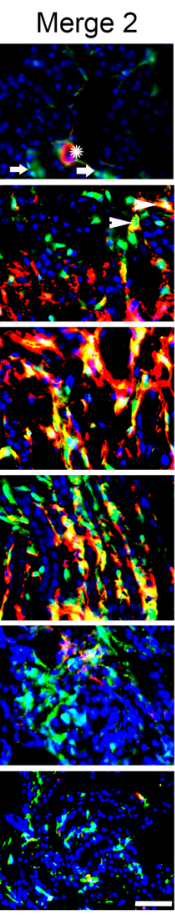

B
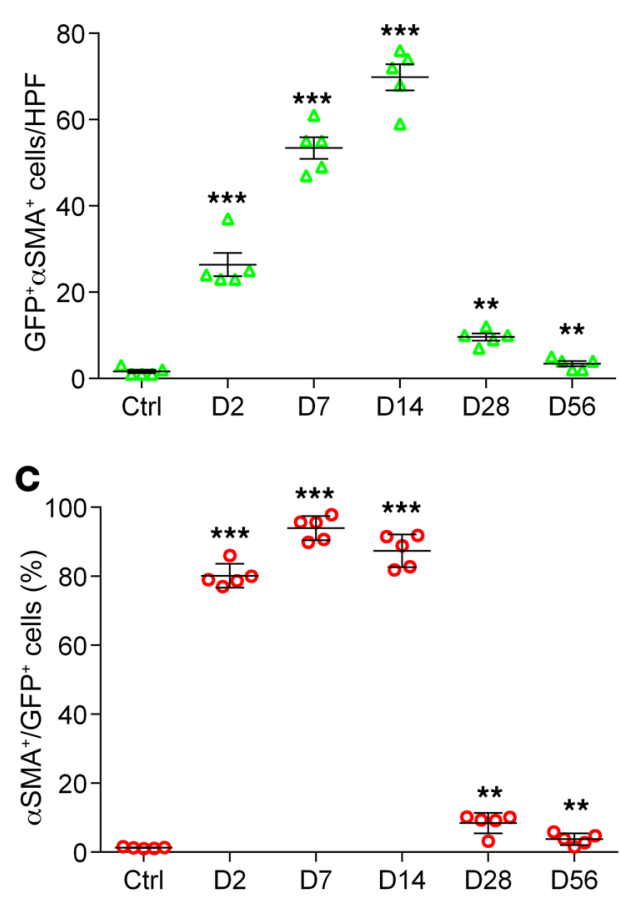

Figure 1. Acute kidney injury induced a transient increase in renal myofibroblasts. (A) Representative images showing Col1a1-GFP+ $\alpha$-smooth mus-

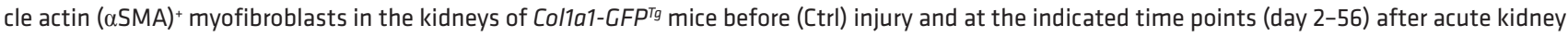
injury (AKI) induced by right nephrectomy (Nx) followed by left ischemia/reperfusion injury (IRI). Arrows, arrowheads, and asterisks indicate Col1a1-GFP ${ }^{+}$ pericytes, Col1a1-GFP+ $\alpha \mathrm{SMA}^{+}$myofibroblasts, and Col1a1-GFP- $\alpha \mathrm{SMA}^{+}$vascular smooth muscle cells, respectively. Scale bar: $25 \mu \mathrm{m}$. Original magnification, $\times 400$. (B) Dot chart showing the cell numbers of Col1a1-GFP+ $\alpha S M A^{+}$myofibroblasts per high-powered field (HPF) at $\times 400$ at the indicated time points.

(C) Dot chart showing the proportion of $\alpha \mathrm{SMA}^{+}$Col1a1-GFP+ myofibroblasts to Col1a1-GFP+ pericytes. Horizontal bars represent the mean, error bars represent the SEM. ${ }^{* *} P<0.01,{ }^{* *} P<0.001$ vs. Ctrl by 1 -way ANOVA with post hoc Dunnett's correction. $n=5$.

of $\alpha \mathrm{SMA}^{+}$myofibroblasts in the acute phase of AKI induced by ischemia/reperfusion injury (IRI) $(4,27)$. Residual focal fibrosis and myofibroblasts noted in functionally recovered kidneys on day 28 after AKI are pathological findings possibly linked to the subsequent CKD transition $(4,28)$. In a progressive renal fibrosis model induced by folic acid, Bechtel et al. demonstrated that the hypermethylation of Rasal1, which encodes an inhibitor of the Ras oncoprotein, leads to perpetuated activation of myofibroblasts and renal fibrosis (8). Our previous study also demonstrated that the hypermethylation of Epo promoter and enhancer in myofibroblasts results in decreased EPO production and anemia in CKD (17). Demethylation by 5 -azacytidine (5-Aza) not only increased Epo expression but also decreased Acta2 expression, suggesting the potential effect of demethylation in the reversal of myofibroblasts to pericytes (17).

Since pericytes participate in tissue repair, microvascular stability, and become scar-producing myofibroblasts in CKD $(7,9,15-24,26)$, we are intrigued by the fate and role of pericytes in AKI. Our findings demonstrated that the activation of pericytes to myofibroblasts in AKI is associated with the hypermethylation of $Y b x 2$, a gene encoding a Y-box-binding protein for Acta 2 repression. During AKD, Ybx 2 methylation maintains pericytes in a nonquiescent phenotype with a higher potential for reactivation by further injuries and to promote indolent CKD progression.

\section{Results}

AKI induced a transient increase of renal myofibroblasts. We induced IRI-AKI in adult male C57BL/6 mice by right nephrectomy (Nx) at the age of 8 weeks followed by left renal IRI 2 weeks later (Supplemental Figure 1A; supplemental material available online with this article; https://doi.org/10.1172/JCI135773DS1). Plasma levels of blood urea nitrogen (BUN) and creatinine increased and peaked on day 2 after IRI-AKI and decreased to comparable levels of Nx control (Ctrl) mice on day 30 (Supplemental Figure 1, B and C). Interestingly, BUN, creatinine, and urine albumin-creatinine ratio (UACR) increased significantly from day 180 (Supplemental Figure 1, B-D). Fibrosis was demonstrated in kidneys on day 30 after IRI-AKI and increased progressively thereafter (Supplemental Figure 1, E and F). Similar to our previous report (4), progressive glomerulosclerosis was also noted. We thus used the IRI-AKI model to study the AKI-CKD transition.

Transgenic Col1a1-GFP reporter mice (Col1a1-GFP ${ }^{T g}$ ) express green fluorescent protein (GFP) in renal pericytes (15). The cell

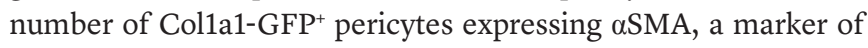
activated pericytes or myofibroblasts, increased markedly after IRI-AKI (Figure 1, A and B). Although $\alpha \mathrm{SMA}^{+} \mathrm{Col1a1}-\mathrm{GFP}^{+}$myofibroblasts decreased after day 14, the cell number on day 56 was still higher than that in Ctrl kidney (Figure 1, A and B), with more than $80 \%$ of Col1a1-GFP ${ }^{+}$pericytes being $\alpha \mathrm{SMA}^{+}$myofibroblasts from day 2 to day 14 after IRI-AKI (Figure 1, A and C). By contrast, 
A

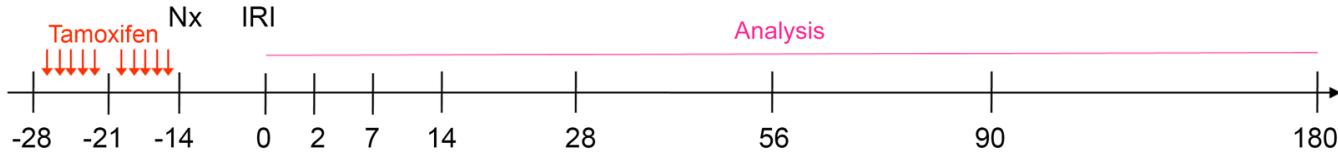

B

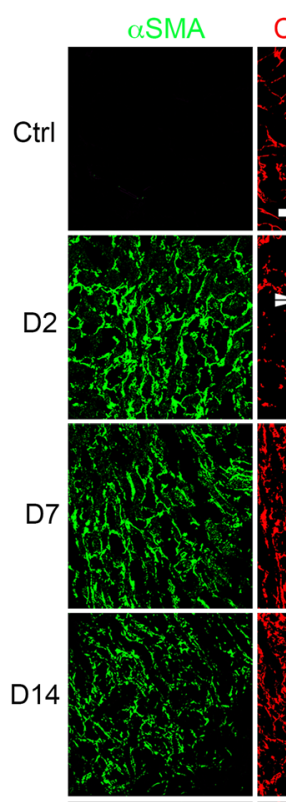

Mice: Col1a2-Cre-ERT $T^{T_{g}}$ ROSA26 $6^{\text {stdtomato/ }}$
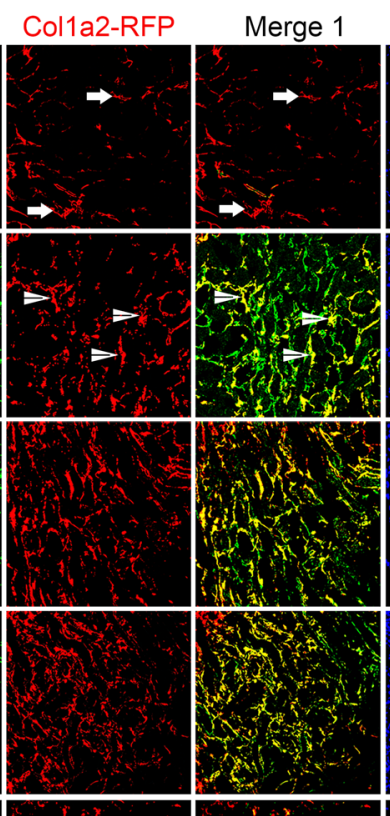

\section{DAPI}
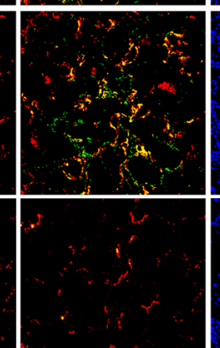

D56

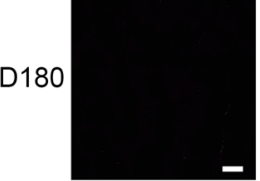

$\mathbf{F}$
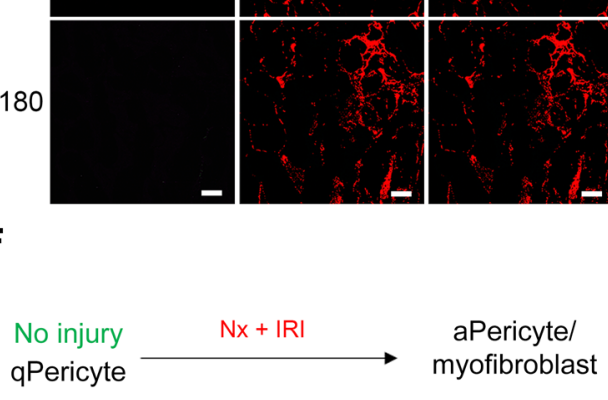
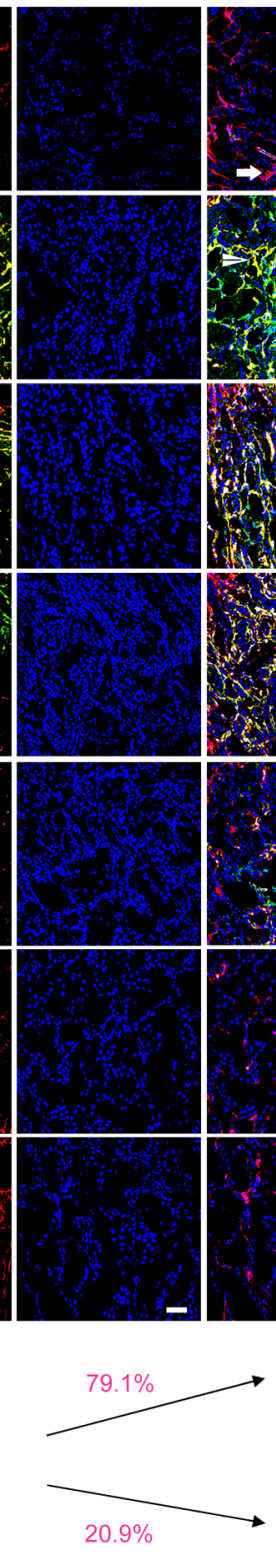

C

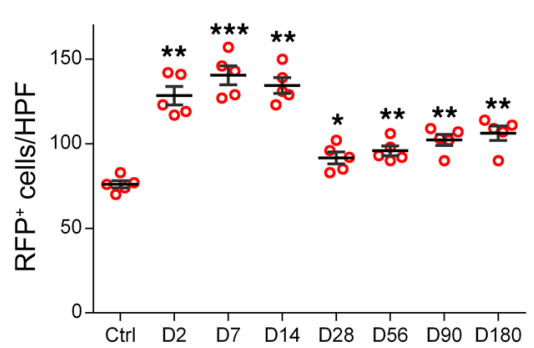

D
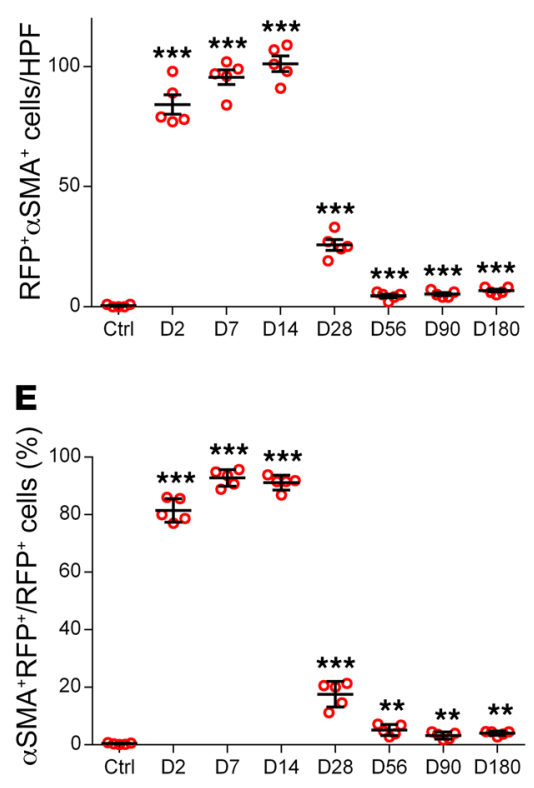

Figure 2. Renal myofibroblasts were derived from pericytes during acute kidney injury. (A) Experimental scheme showing cohort labeling by tamoxifen and AKI-induced by Nx + IRI (IRI-AKI) in Col1a2-CreERT $T^{T_{g}}$ ROSA26 fstdTomato/+ mice. Analyses were performed at the indicated time points. (B) Representative images showing the Col1a2-RFP+ pericyte lineage and $\alpha \mathrm{SMA}^{+}$Col1a2-RFP+ ${ }^{+}$yofibroblasts in the kidneys. Arrows and arrowheads indicate Col1a2-RFP+ pericytes and $\alpha \mathrm{SMA}^{+}$Col1a2-RFP+ myofibroblasts, respectively. Scale bars: $25 \mu \mathrm{m}$. Original magnification, $\times 400$. (C) Dot chart showing the cell numbers of the Col1a2-RFP+ pericyte lineage/HPF at the indicated time points. (D) Dot chart showing the cell numbers of $\alpha \mathrm{SMA}^{+} \mathrm{Col}^{+} \mathrm{a2}-\mathrm{RFP}^{+}$myofibroblasts/HPF. (E) Dot chart showing the proportion of $\alpha \mathrm{SMA}^{+}$Col1a2-RFP+ myofibroblasts to Col1a2-RFP+ pericytes. (F) AKI induced the activation of qPericytes into aPericytes. Based on the fate of Col1a2-RFP+ pericytes (Supplemental Figures 4-6), aPericytes might undergo apoptosis or inactivation (iPericytes) by day 28 after IRI-AKI. Horizontal bars represent the mean, error bars represent the SEM. ${ }^{*} P<0.05,{ }^{* *} P<0.01,{ }^{* * *} P<0.001$ vs. Ctrl by 1 -way ANOVA with post hoc Dunnett's correction. $n=5$. 
A
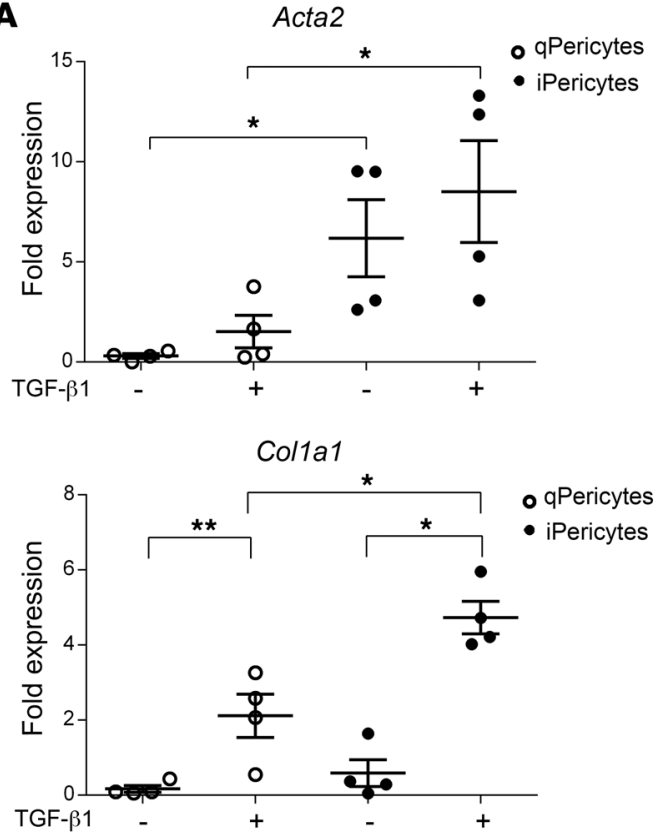
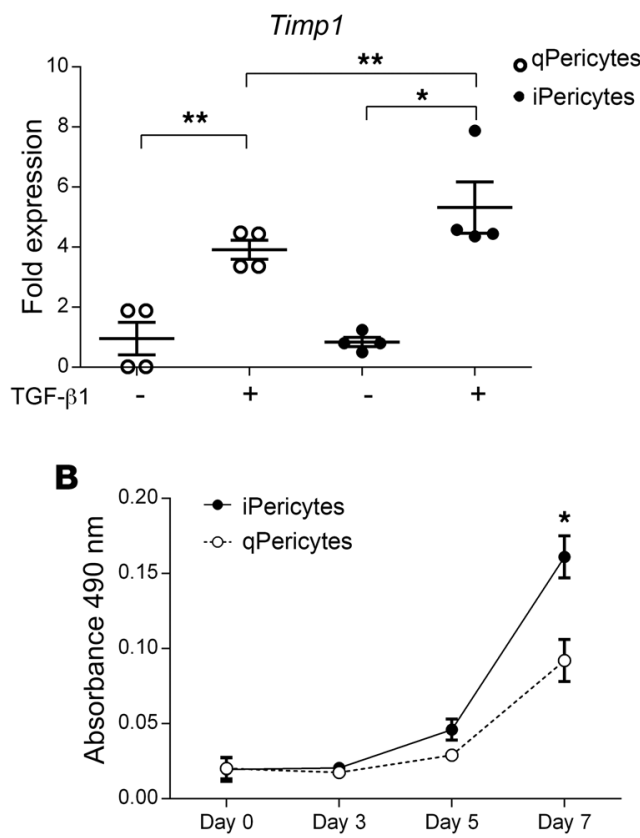

Figure 3. Inactivated pericytes retained a higher potential for fibrosis and cell proliferation in vitro. (A) Primary cultures of qPericytes and iPericytes from the kidneys before and on day 28 after IRI-AKI, respectively, were exposed to TCF- $\beta 1$ or Ctrl culture medium for 16 hours. Dot charts showed the expression of Acta2, Col1a1, and Timp1 assessed by quantitative PCR. The expression levels were normalized by Gapdh. Horizontal bars represent the mean, error bars represent the SEM. ${ }^{*} P<0.05$, ${ }^{*} P<0.01$ by 1-way ANOVA with post hoc Tukey's correction. $n=4$ for each group. (B) Line chart showing the cell proliferation of qPericytes and iPericytes assessed by 3-(4,5-Dimethylthiazol-2-yl)-2,5-diphenyltetrazolium bromide (MTT) assays. Data are expressed as the mean \pm SEM. ${ }^{*} P<0.05$ by $t$ test at each time point. $n=4$ for each group.

tubular epithelial cells enclosed by laminin ${ }^{+}$basement membrane, $\mathrm{CD}^{+} 1^{+}$endothelial cells, and $\mathrm{F} 4 / 80^{+}$macrophages did not coexpress Col1a1-GFP or $\alpha$ SMA (Supplemental Figure 2). In parallel with the transient increase in the number of $\alpha \mathrm{SMA}^{+} \mathrm{Col1a1-GFP}{ }^{+}$ myofibroblasts, the proportion of $\alpha \mathrm{SMA}^{+} \mathrm{Col1a} 1-\mathrm{GFP}^{+}$myofibroblasts to Col1a1-GFP ${ }^{+}$pericytes also decreased markedly from day 28 (Figure 1, A and C), suggesting that the activated pericytes/ myofibroblasts underwent inactivation during renal recovery.

Although more than $80 \%$ of Col1a1-GFP ${ }^{+}$pericytes coexpressed the myofibroblast marker $\alpha \mathrm{SMA}$ from day 2 to day 14 after IRI-AKI (Figure 1, A and C), Col1a1-GFP was a dynamic marker whose detection relied on the active expression of the Col1a1 gene (15). To unbiasedly determine the contribution of pericytes to myofibroblasts and their fate after AKI, we generated Colla2-CreERT Tg ROSA $26^{\text {fstdomato/+ }}$ mice and permanently labeled Col1a2-expressing pericytes with red fluorescence protein (RFP) by tamoxifen. Although only $60 \%$ of Col1a1-GFP ${ }^{+}$pericytes coexpressed Col1a2-RFP, all Col1a2-RFP ${ }^{+}$cells were Col1a1-GFP ${ }^{+}$pericytes in Col1a1-GFP ${ }^{T g}$ Col1a2-CreERT ${ }^{T g}$ ROSA26 $6^{\text {fstdomato/+ }}$ mice (Supplemental Figure 3, A-C) and we could trace the fate of genetically labeled Col1a2-RFP ${ }^{+}$pericytes after AKI in the following experiments. Similar to the findings in Col1a1-GFP ${ }^{T g}$ mice (Figure 1), the cell numbers of Col1a2-RFP ${ }^{+}$pericytes and $\alpha \mathrm{SMA}^{+} \mathrm{Col1a} 2-\mathrm{RFP}^{+}$ myofibroblasts in the kidneys of Col1a2-CreERT ${ }^{T g}$ ROSA2 $6^{\text {fstdTomato/+ }}$ mice increased after IRI-AKI and more than $80 \%$ of Col1a2-RFP ${ }^{+}$ pericytes coexpressed $\alpha$ SMA from day 2 to day 14 (Figure 2, A-E). According to the staining of terminal deoxynucleotidyl transferase dUTP nick end labeling (TUNEL) and Ki67 in kidneys before (Ctrl) and after IRI-AKI, both cell apoptosis and proliferation of
Col1a2-RFP ${ }^{+}$pericytes were detected soon after IRI-AKI (Supplemental Figures 4-6). In agreement with the finding from TUNEL staining, we also detected apoptotic pericytes by the colocalization of cleaved caspase-3 and Col1a2-RFP (Supplemental Figure 7). $\mathrm{TUNEL}^{+}$Col1a2-RFP ${ }^{+}$pericytes predominated after day 7 of IRI-AKI, suggesting that myofibroblasts underwent apoptosis and the cell numbers decreased during renal recovery (Supplemental Figure 6). It was noteworthy that cell numbers of Col1a2-RFP ${ }^{+}$ pericytes and $\alpha \mathrm{SMA}^{+} \mathrm{Col1a} 2-\mathrm{RFP}^{+}$myofibroblasts did not return to the numbers found in the Ctrl kidneys and increased after day 56 (Figure 2, B-D). Though the cell numbers of $\alpha \mathrm{SMA}^{+} \mathrm{Col1a} 2-\mathrm{RFP}^{+}$ myofibroblasts continued to be higher than those in the Ctrl kidneys, the proportion of $\alpha \mathrm{SMA}^{+} \mathrm{Col1a} 2-\mathrm{RFP}^{+}$myofibroblasts to Col1a2-RFP ${ }^{+}$pericytes decreased significantly from day 28 (Figure $2, \mathrm{~B}, \mathrm{D}$, and $\mathrm{E}$ ), corresponding to the suggestion above that activated pericytes/myofibroblasts underwent inactivation during renal recovery (Figure $1, \mathrm{~A}$ and $\mathrm{C}$ ).

Myofibroblasts underwent inactivation during renal recovery from AKI. To study the role of pericytes in the AKI-CKD continuum, we performed a whole-genome microarray of kidney pericytes isolated from different time points before and after IRI-AKI. Heatmap and principal component analysis (PCA) demonstrated distinctive gene expression patterns of pericytes isolated from the indicated time points (Supplemental Figure 8). Hence, we defined the pericytes isolated from different time points as being in the quiescent (qPericytes, normal kidney), activated (aPericytes or myofibroblasts, AKI on day 7), and inactivated (iPericytes, AKD on day 28) status, respectively. According to the cell numbers of Col1a2- $\mathrm{RFP}^{+}$pericytes in Figure 2C, the fate of aPericytes includ- 
A

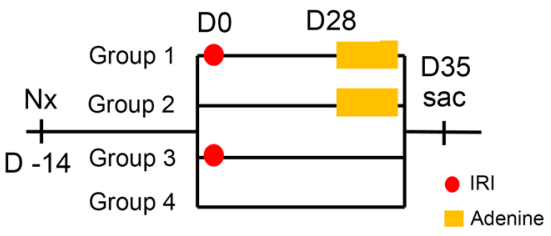

C

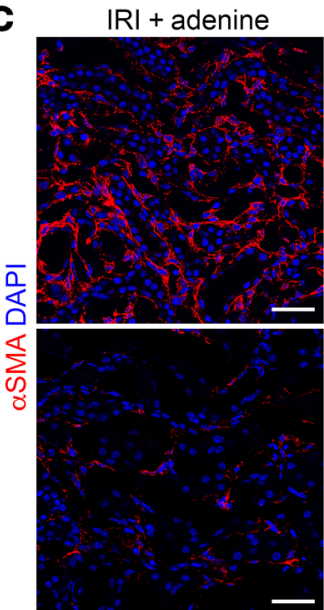

IRI

E

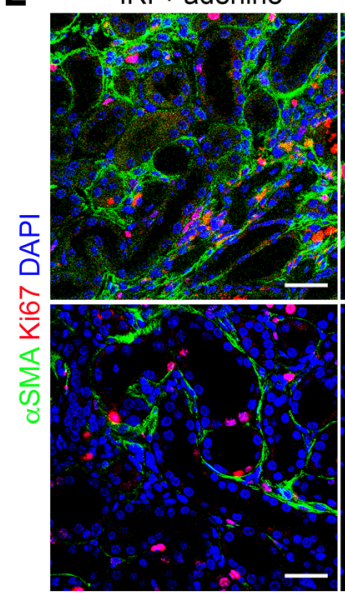

$|R|$

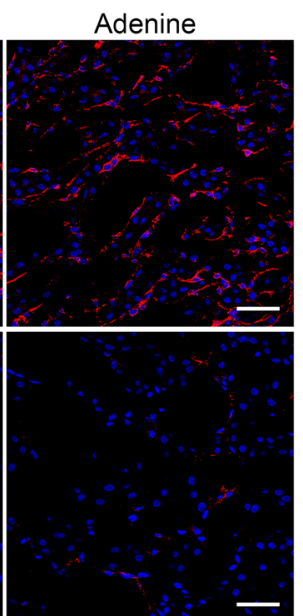

Ctrl

Adenine

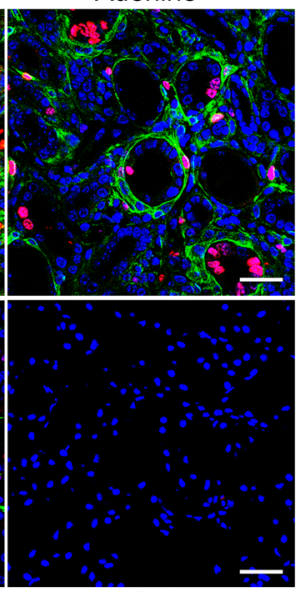

Ctrl
B
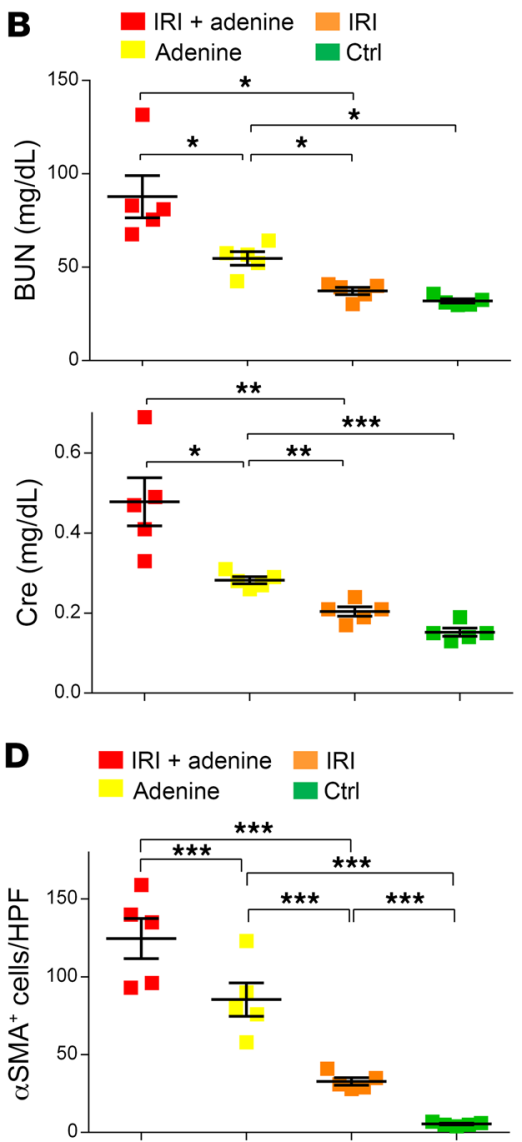

F $\square$ IRI + adenine $\square$ IRI

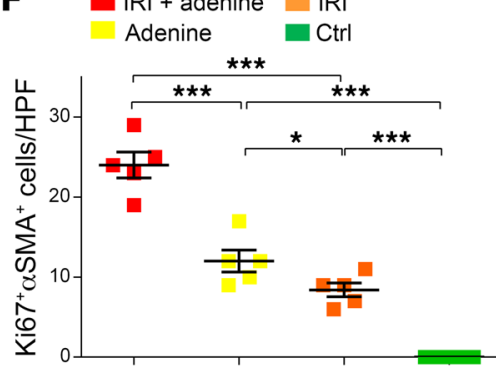

Figure 4. Inactivated pericytes retained a higher potential for cell proliferation induced by a second injury in vivo. (A) Experimental scheme of a second AKI on the kidneys that recovered from the first AKI. IRI-AKI was induced on day 0 and allowed to recover until the second $\mathrm{AKI}$ induced by 1-week adenine diet from day 28. Sham operation on day 0 and regular diet from day 28 served as the Ctrl. The mice were allocated to group 1 (IRI+adenine, $A K I$ on kidneys that recovered from the first $A K I)$, group 2 (adenine, single $A K I)$, group 3 (IRI, single $A K I)$, and group 4 (Nx only, CtrI). (B) Dot charts showing the plasma levels of BUN and Cre. (C) Representative images showing $\alpha \mathrm{SMA}^{+}$myofibroblasts in the kidneys. Scale bars: $25 \mu \mathrm{m}$. Original magnification, $\times 400$. (D) Dot chart showing the cell numbers of $\alpha \mathrm{SMA}^{+}$myofibroblasts/HPF at $\times 400$ in the renal sections. (E) Representative images showing $\mathrm{Ki}_{67}{ }^{+} \alpha \mathrm{SMA}^{+}$ myofibroblasts in the kidneys. Scale bars: $25 \mu \mathrm{m}$. Original magnification, $\times 400$. (F) Dot chart showing the cell numbers of $\mathrm{Ki}^{6} 7^{+} \alpha \mathrm{SMA}^{+}$myofibroblasts/HPF in the renal sections. Horizontal bars represent the mean, error bars represent the SEM ${ }^{*} P<0.05,{ }^{* *} P<0.01,{ }^{* *} P<0.001$ by 1 -way ANOVA with post hoc Tukey's correction. $n=5$ for each group. ed apoptosis (79.1\%) and inactivation (20.9\%) during renal recovery from AKI (Figure $2 \mathrm{~F}$ ). We also found that renal expression of platelet-derived growth factor-B and transforming growth factor- $\beta 1$ (TGF- $\beta 1$ ), 2 key growth factors for pericyte proliferation and activation $(7,16)$, were transiently increased, providing a possible explanation for the change in pericyte number after AKI (Supplemental Figure 9).

iPericytes retained a higher potential for reactivation by injuries. We cultured qPericytes and iPericytes isolated from normal and day 28 AKD kidneys, respectively. Though the proportion of $\alpha \mathrm{SMA}^{+} \mathrm{Col1a} 2-\mathrm{RFP}^{+}$aPericytes to all Col1a2-RFP ${ }^{+}$pericytes decreased significantly from day 28 after AKI (Figure 2, B and E), the expression levels of Acta 2 in iPericytes were higher than those in qPericytes (Figure 3A). In addition, iPericytes showed a higher profibrotic potential as evidenced by elevated expression levels of Acta2, Col1a1, and Timp1 induced by TGF- $\beta 1$ (Figure 3A). Moreover, iPericytes showed a higher proliferative potential (Figure
3B). One month after the first IRI-AKI, mice showed higher BUN and creatinine and more myofibroblasts when they were subjected to a second AKI induced by adenine (adenine-AKI) (Figure $4, \mathrm{~A}-\mathrm{D})$. Increased cell proliferation was demonstrated in renal $\alpha \mathrm{SMA}^{+}$myofibroblasts of mice subjected to adenine-AKI 1 month after functional recovery from IRI-AKI (Figure 4, E and F). Together, these data demonstrated that renal iPericytes retained a higher potential for reactivation, signifying their profibrotic role in functionally recovered kidneys subjected to a second AKI.

Demethylation by 5-azacytidine during AKI attenuated CKD progression after renal recovery. Because iPericytes have a higher potential for reactivation than qPericytes, epigenetic modification was studied. We found increased expression of DNA methyltransferase 1 (DNMT1) in Col1a2- $\mathrm{RFP}^{+}$aPericytes (Supplemental Figure 10A) and upregulation of renal DNMT1 protein after IRI-AKI (Supplemental Figure 10, B and C). In addition, the expression levels of renal Dnmt1, Dnmt3a, and Dnmt3b were upregulated 
A
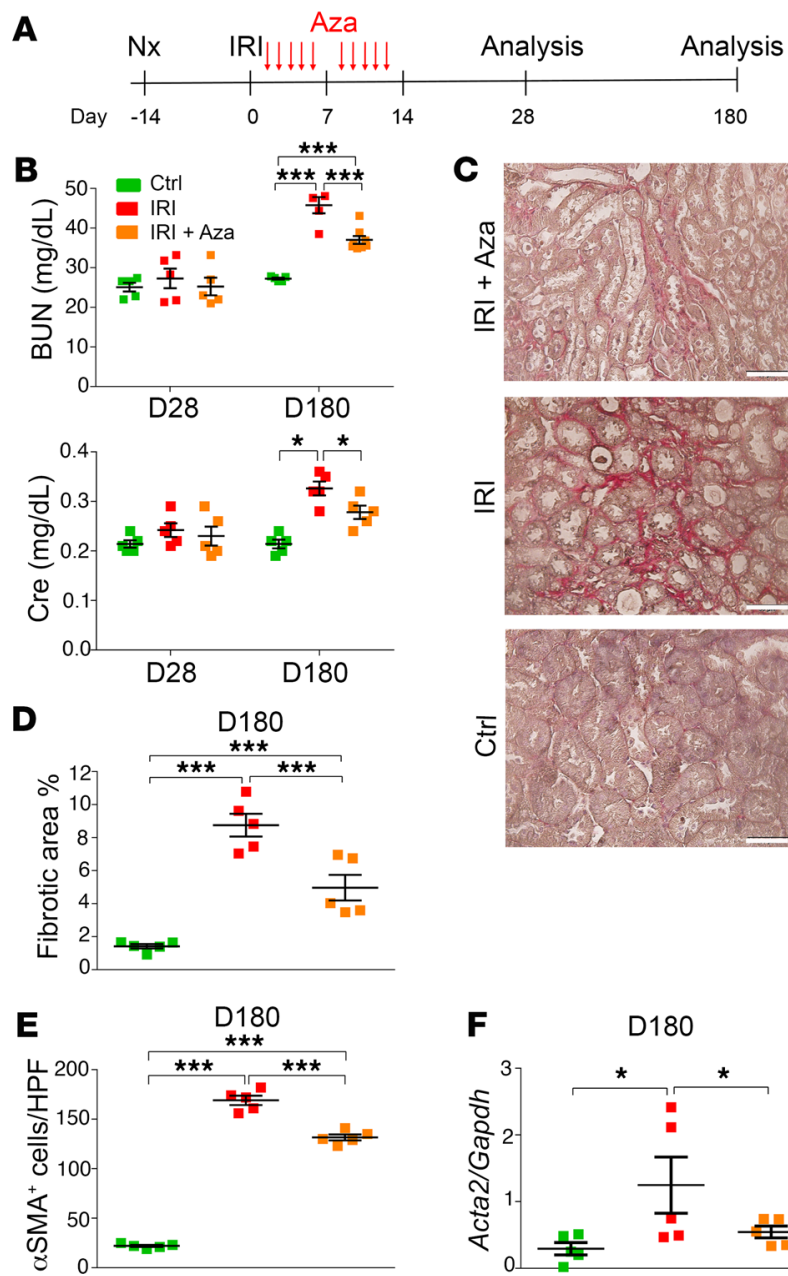
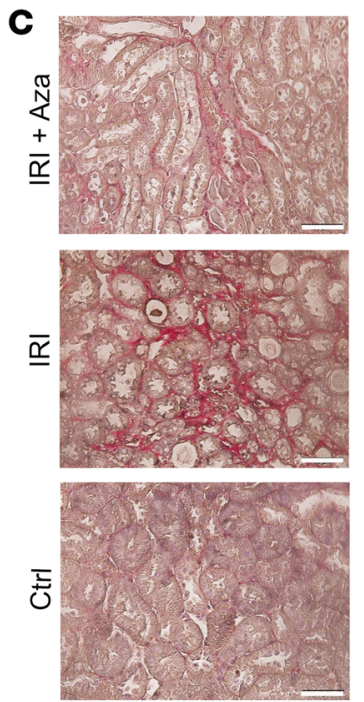

$\mathbf{F}$

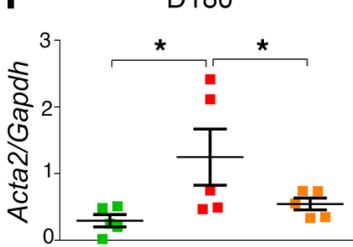

after injury (Supplemental Figure 10D). To gain insight into the role of DNA methylation in the AKI-CKD transition, mice were administered with 5-Aza or vehicle for 2 weeks after IRI or sham operation and analyzed on day 28 and day 180 (Figure 5A). The levels of BUN and creatinine were similar between the groups on day 28 , but both increased significantly in mice on day 180 after IRI-AKI (Figure 5B). AKI mice treated with 5-Aza showed lower increases in BUN and creatinine on day 180 (Figure 5B). Pathologic study revealed increased Picrosirius red-stained fibrotic area and $\alpha \mathrm{SMA}^{+}$myofibroblasts in kidneys after IRI-AKI, both attenuated by 5-Aza treatment (Figure 5, C-E). The reduction of renal Acta2 expression further confirmed the preventive effect of 5-Aza treatment (Figure 5F). These data suggested the impact of epigenetic modification by methylation during AKI on the subsequent development of CKD.

5-azacytidine reversed aPericytes to become quiescent. To study how the changes in DNA methylation during AKI affects the response of recovered kidneys to a second AKI, we treated mice with 5-Aza or vehicle after IRI-AKI and then induced the second AKI by adenine on day 28 (Figure 6A). Nx only and regular diet served as the Ctrl. The plasma levels of BUN and creatinine were higher in the repeated AKI group (IRI+adenine) than those of the single AKI group induced by either IRI or adenine (Figure 6, B and C). No difference in BUN and creatinine was found between the

Figure 5. Demethylation by 5-azacytidine during acute kidney injury attenuated the progression to chronic kidney disease. (A) Experimental scheme for the IRI-AKI and 5-azacytidine (Aza) treatment. Nx followed by sham operation served as the Ctrl. Plasma BUN and Cre were analyzed on day 28 and day 180 . Kidneys were analyzed on day 180 . (B) Dot charts showing the plasma BUN and Cre in each group. (C) Representative images showing Picrosirius red staining in the renal sections on day 180 . Scale bars: $50 \mu \mathrm{m}$. (D) Dot chart showing the quantification of Picrosirius redstained fibrotic area in the renal sections. (E) Dot chart showing the cell numbers of $\alpha \mathrm{SMA}^{+}$myofibroblasts/HPF in the renal sections. (F) Dot chart showing the renal Acta2 expression normalized by Gapdh. Horizontal bars represent the mean, error bars represent the SEM. ${ }^{*} P<0.05$, ${ }^{* *} P<0.001$ by 1-way ANOVA with post hoc Tukey's correction. $n=5$ for each group.

IRI-AKI and Ctrl groups, demonstrating functional recovery from the first IRI-AKI (Figure 6, B and C). Compared with the repeated AKI group treated by vehicle, the group with 5-Aza treatment showed fewer $\alpha \mathrm{SMA}^{+}$myofibroblasts and $\mathrm{Ki}^{+} \mathrm{7}^{+} \alpha \mathrm{SMA}^{+}$myofibroblasts, less Picrosirius red-stained fibrotic area, and lower profibrotic gene expression, but had no change on the levels of BUN and creatinine induced by the second adenine-AKI (Figure 6, B-J). These data showed that demethylation during AKI could attenuate the profibrotic response but did not affect the functional abnormality induced by repeated or a second AKI.

We cultured aPericytes from kidneys on day 7 after AKI and stimulated them with TGF- $\beta 1$ after 3-day exposure to 5-Aza or vehicle (Supplemental Figure 11A). qPericytes isolated from normal kidneys served as Ctrl. After 5-Aza treatment, the baseline expression of Acta2 in aPericytes was reduced to the level shown in qPericytes, the upregulation of Acta 2 by TGF- $\beta 1$ was also reduced (Supplemental Figure 11B). The levels of baseline and TGF- $\beta 1^{-}$ induced expression of Colla1 in aPericytes were also reduced after 5-Aza treatment (Supplemental Figure 11C).

Since 5-Aza could decrease the expression of the activation marker Acta2 in aPericytes, we then studied whether 5-Aza could affect the function of aPericytes for vascular stabilization in 3D collagen gels (Figure 7A). Capillary network formation was demonstrated 24 hours after coculture of pericytes and human umbilical vein endothelial cells (HUVECs) in the collagen gel (Figure 7, B and C). At baseline, the capillary density was lower in HUVECs alone and coculture with aPericytes (Figure 7D). Coculture with qPericytes but not aPericytes could retard kallikreininduced capillary tube regression and gel retraction (Figure 7, E and F). However, aPericytes after 5-Aza treatment resumed their function for vascular stabilization (Figure 7, E and F). qPericytes expressed higher levels of microvascular stabilizing and angiogenic factors including Timp3, Angpt1, and Angpt2, which encoded tissue inhibitor of metalloproteinase-3 (TIMP3), angiopoietin-1, and angiopoietin-2, respectively (Figure 7G). Treatment with 5-Aza reversed the repression of these vascular stabilizing factors in aPericytes, a mechanism supporting the recovery of function for vascular stabilization. These data collectively demonstrated that demethylation by 5 -Aza could reverse aPericytes to become more similar to qPericytes.

Ybx2 and Acta2 showed the opposite expression patterns in pericytes after AKI. In our whole-mouse genome microarray, we identified substantial changes in the expression of 246 genes in pericytes after AKI. Among these genes, we focused on Ybx2 

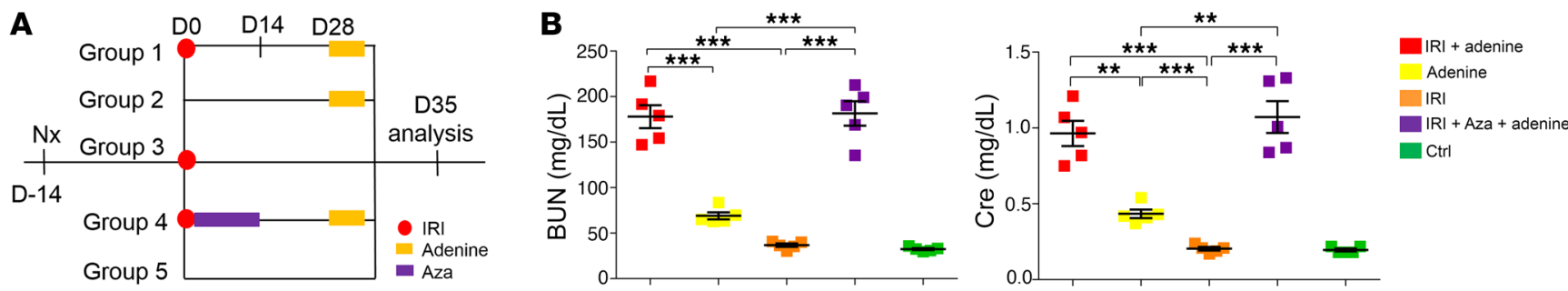

C
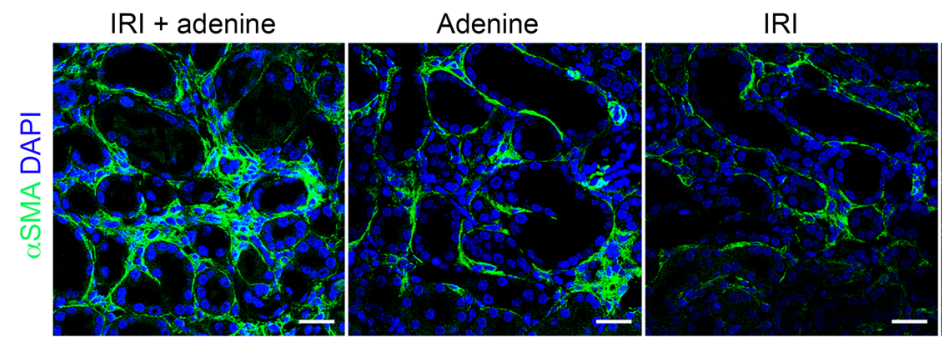

IRI + Aza + adenine

Ctrl

D
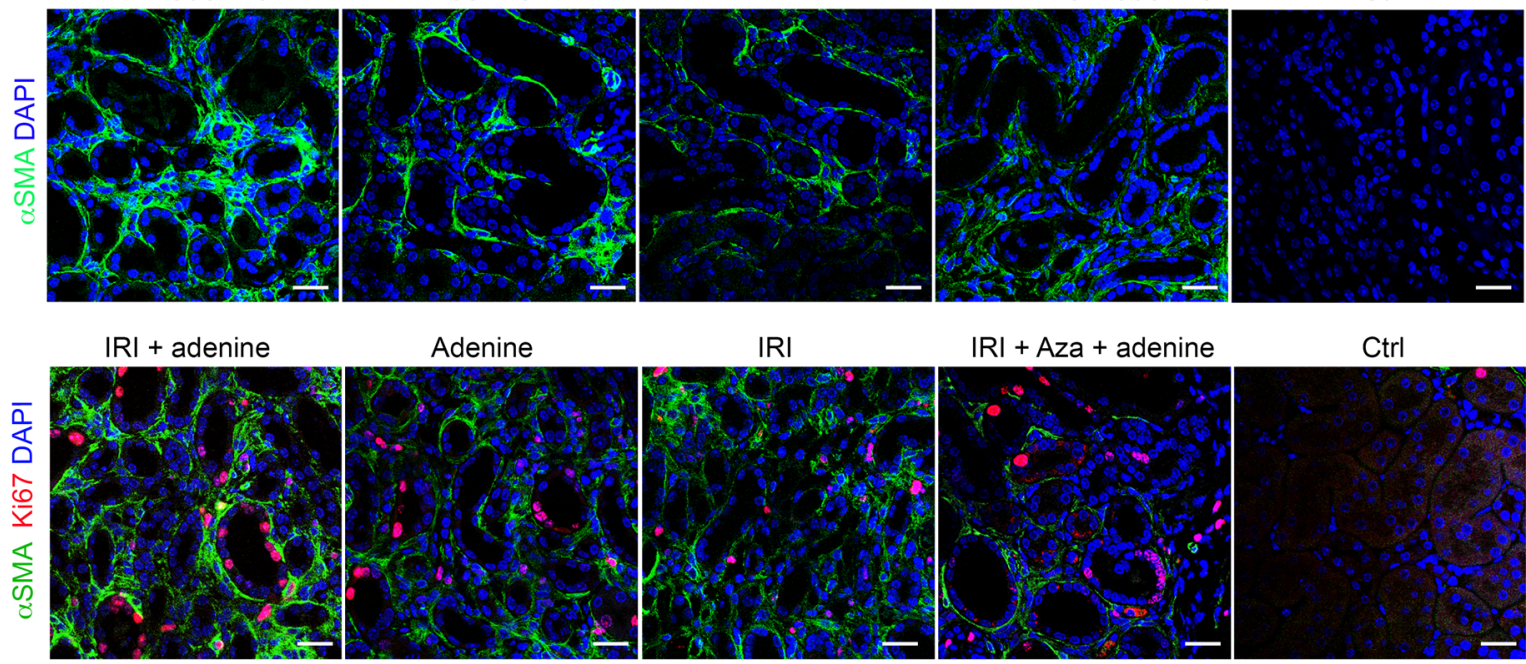

E

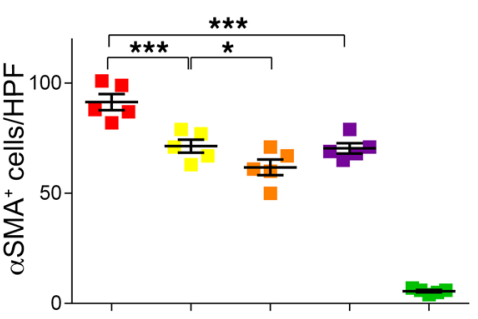

H

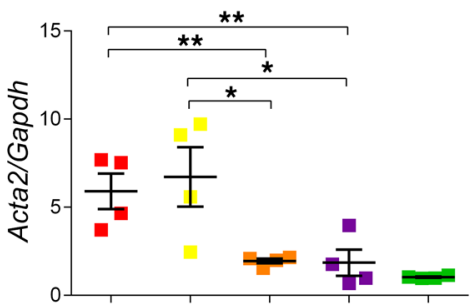

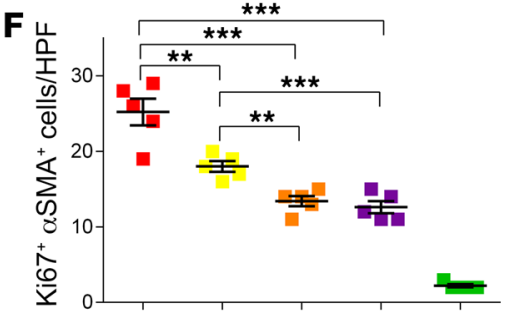

G

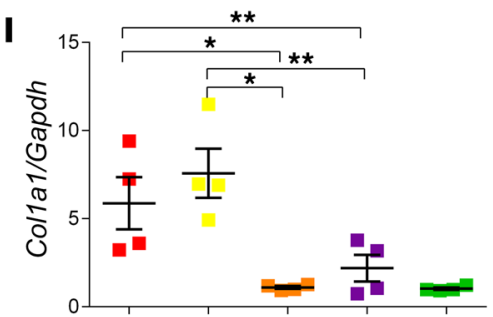

IRI + Aza + adenine

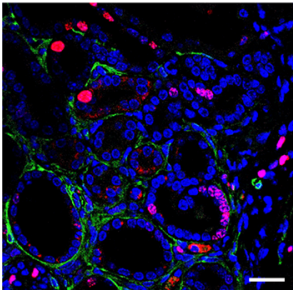

Ctrl

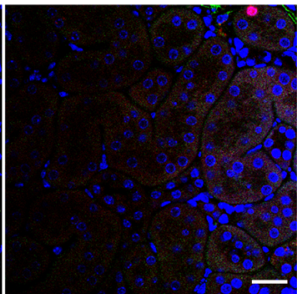

Figure 6. Demethylation by 5-azacytidine during acute kidney injury attenuated the reactivation of pericytes and renal fibrosis induced by a second injury. (A) Experimental scheme of adenine-AKI on the kidneys that recovered from the first IRI-AKI. The protocol for the administration of Aza or vehicle is shown in Figure 5A. Sham operation on day 0 and regular diet from day 28 served as the Ctrl. The mice were allocated to group 1 (IRI + adenine), group 2 (adenine only), group 3 (IRI only), group 4 (IRI + Aza + adenine), and group 5 (Nx only, Ctrl). Analyses were performed on day 35 . (B) Dot charts showing the plasma BUN and Cre. $n=5$. (C) Representative images showing $\alpha S M A^{+}$myofibroblasts in the renal sections. Scale bars: $25 \mu \mathrm{m}$. Original magnification, $\times 400 . n=5$. (D) Representative images showing Ki67 ${ }^{+} \alpha S M A^{+}$myofibroblasts in the kidneys. Scale bars: $25 \mu \mathrm{m}$. Original magnification, $\times 400$. $n=5$. (E) Dot chart showing the cell numbers of $\alpha S M A^{+}$myofibroblasts/HPF in the renal sections. $n=5$. (F) Dot chart showing the cell numbers of Ki67 ${ }^{+} \alpha S M A^{+}$myofibroblasts in the renal sections. $n=5$. (G) Dot chart showing the quantification of Picrosirius red-stained fibrotic area in the renal sections. $n=5$. (H-J) Dot chart showing the expression of renal Acta2, Col1a1, and Col3a1. Horizontal bars represent the mean, error bars represent the SEM. ${ }^{*} P<0.05,{ }^{* *} P<0.01$, ${ }^{* * *} P<0.001$ by 1 -way ANOVA with post hoc Tukey's correction. $n=4$ for each group.

because it was a plausible repressor of Acta2 in qPericytes. YBX2 could be identified in the Ctrl kidneys by immunohistochemistry, especially in interstitial cells (Figure 8A and Supplemental Figure 12A). Col1a1-GFP ${ }^{+}$pericytes expressed YBX2 in the Ctrl kidneys of Col1a1-GFP $P^{T g}$ mice (Figure 8B and Supplemental Figure 12B). Interestingly, YBX2 staining increased in injured tubular epithelial cells after IRI-AKI but decreased in interstitial cells (Figure $8 \mathrm{C}$ and Supplemental Figure 12A). The renal expression of $Y b x 2$ mRNA 
A

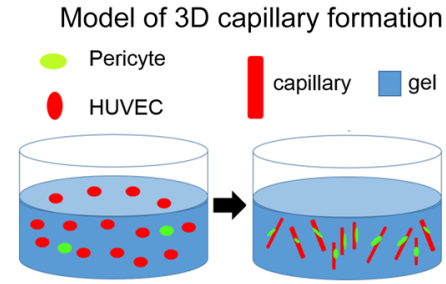

D

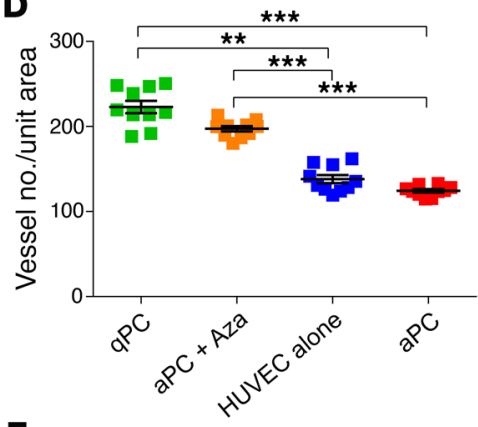

F

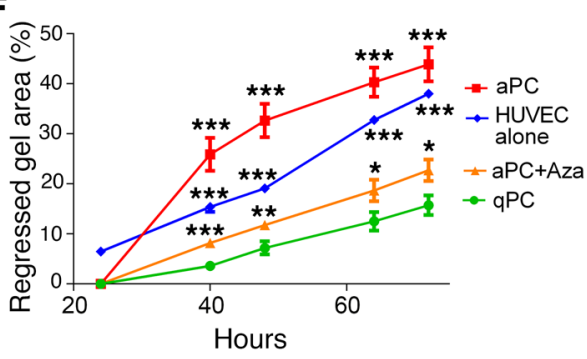

G

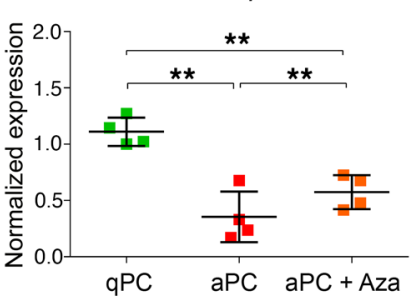

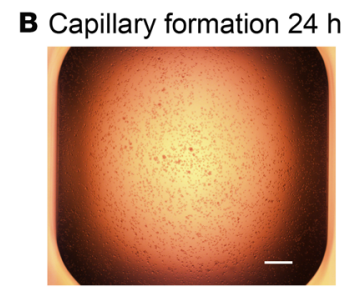

c

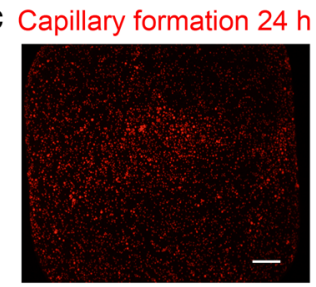

E
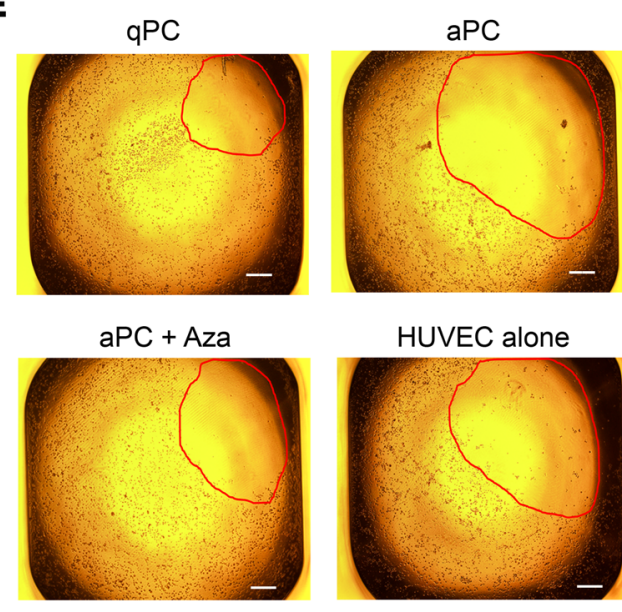

HUVEC alone
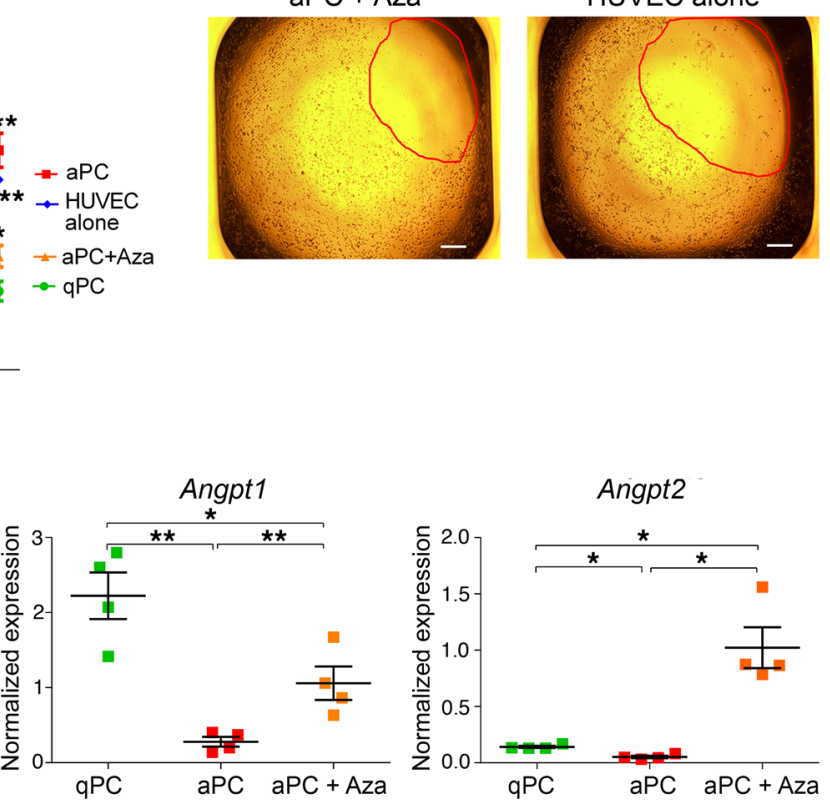

Figure 7. Demethylation by 5-azacytidine reversed the vascular stabilizing function of myofibroblasts. (A) The scheme showing the model of $3 \mathrm{D}$ capillary formation by mixing pericytes (green) and HUVECs (red) in collagen gels. (B) A representative bright-field image demonstrating the cross sections of capillary tubes 24 hours after mixing pericytes and HUVECs in collagen gels. Scale bars: $250 \mu \mathrm{m}$. Original magnification, $\times 4$. (C) A representative immunofluorescent image showing capillary formation (red) 24 hours after mixing the pericytes and CellTracker Red-stained HUVECs in collagen gels. Scale bars: $250 \mu \mathrm{m}$. Original magnification, $\times 4$. (D) Dot chart showing the vascular density in the collagen gels in the presence of coculture with qPericytes (qPC), aPericytes (aPC, myofibroblasts), aPericytes after Aza treatment for 3 days (aPC + Aza), or in the absence of pericyte coculture (HUVEC alone) for 24 hours. $n$ $=10$. (E) Images showing the collapse of collagen gels induced by kallikrein (KLK) in gels with HUVECs in the presence or absence of pericyte coculture. Scale bars: $250 \mu \mathrm{m}$. Original magnification, $\times 4$. (F) Line chart showing the percentage of collapsed area in gels with HUVECs in the presence or absence of pericyte coculture at the indicated time points after KLK administration. $n=10$. (G) Dot charts showing the expression of Timp3, Angpt1, and Angpt2 assessed by quantitative PCR. The expression levels were normalized to Gapdh. Horizontal bars represent the mean, error bars represent the SEM. $n=4 .{ }^{*} P<0.05,{ }^{*} P$ $<0.01,{ }^{* * *} P<0.001$ by 1 -way ANOVA with post hoc Tukey's correction. was not different between Ctrl and IRI-AKI (Figure 8D), but quantitative PCR showed that $Y b x 2$ mRNA decreased in aPericytes on day 7 after IRI-AKI and recovered partially in iPericytes on day 28 (Figure $8 \mathrm{E}$ ). In contrast, Acta 2 mRNA in pericytes showed the opposite trend (Figure $8 \mathrm{~F}$ ).

The promoter of the Acta2 repressor Ybx2 was methylated in pericytes after AKI. TGF- $\beta 1$ exposure decreased Ybx2 mRNA in the primary culture of qPericytes (Figure 9A). The expression of $Y b x 2$ bounced back upon the removal of TGF- $\beta 1$ after 24-hour exposure; however, the expression of $Y b x 2$ did not return upon the removal of TGF- $\beta 1$ after 5-day exposure (Figure 9A). Methylated DNA immunoprecipitation (MeDIP) revealed the methylation of $Y b x 2$ promoter in qPericytes after exposure to TGF- $\beta 1$ for 5 days (Figure 9B). In aPericytes isolated from kidneys on day 7 after IRI-AKI, MeDIP confirmed the methylation of $Y b x 2$ (Supplemental Figure 13). Bisulfite genomic sequencing confirmed the hypermethylation of the $Y b x 2$ promoter in aPericytes (Supplemental Figure 14). Moreover, we also demonstrated the methylation of Tead1, Pura, and Ybx1, known Acta2 repressors (29-32). The methylation of Tead1, Pura, Ybx1, and $Y b x 2$ in aPericytes was reduced after 5-Aza treatment (Supplemental Figure 13). Consistently, the expression levels of $Y b x 1, Y b x 2$, Tead1, and Pura mRNAs were higher in qPericytes than those in aPericytes (Supplemental Figure 15). Demethylation by 5-Aza partially recovered the expression of these genes in aPericytes.

We then designed 3 pairs of primers to amplify different promoter regions of the Acta2 gene (Figure 9C). By chromatin immunoprecipitation-polymerase chain reaction (ChIP-PCR), we identified that the promoter regions of Acta2 were bound by YBX2 protein (Figure 9D). In addition to suppressing $Y b x 2$ transcription (Figure 9A), TGF- $\beta 1$ exposure also decreased the binding of YBX2 to the Acta2 promoter (Figure 9D). We then demonstrated that lentiviral overexpression of YBX2 decreased $\alpha$ SMA expression in aPericytes (Figure 9, E and F). These data collectively demonstrated that $Y b x 2$ methylation in pericytes led to a higher potential for reactivation.

\section{Discussion}

We report 4 important findings in the fate and role of pericytes after AKI: (a) pericytes are the major progenitor cells of renal 

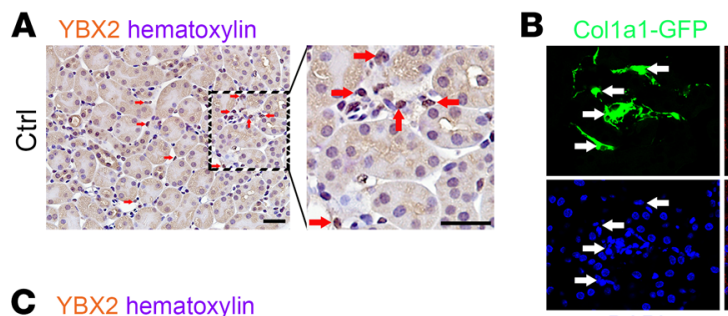

DAPI
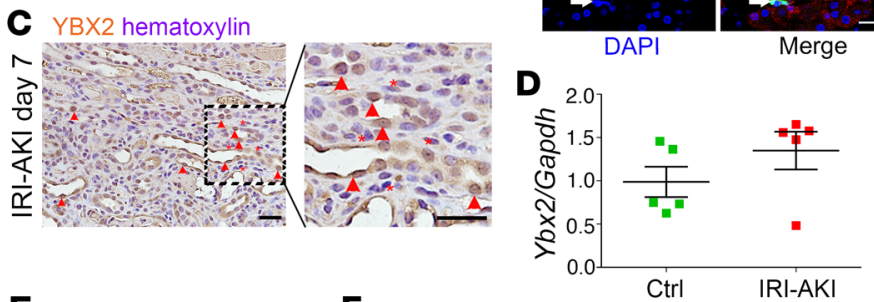
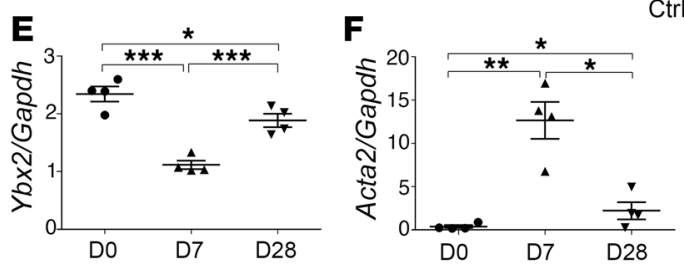

myofibroblasts after AKI through the activation of $\alpha \mathrm{SMA}$ and cell proliferation; (b) apoptosis lowers the number of myofibroblasts during renal recovery, but the cell number and activation potential do not return to the quiescent baseline due to hypermethylation of Acta2 repressor genes such as Ybx2; (c) Ybx2 hypermethylation maintains pericytes in an inactivated, nonquiescent phenotype, which is prone to reactivation upon a second AKI; (d) demethylation by 5-Aza switches myofibroblasts back to a quiescent status with the function of vascular stabilization.

Kidney pericytes are named when they are embedded within the microvascular basement membrane and in close contact with endothelial cells $(15,22,24)$. When these cells are not in direct contact with endothelial cells, they are called perivascular fibroblasts (15). Pericytes have been demonstrated as the major source of scar-producing myofibroblasts in CKD with progressive fibrosis $(15,22,24)$. Primary myofibroblasts isolated from fibrotic kidneys remain activated even when cultured in vitro (33). In line with our findings in AKI, hypermethylation of the Rasal1 gene leads to the perpetuation of myofibroblast activation and fibrogenesis in CKD (8). However, hypermethylation of the Epo gene in myofibroblasts leads to decreased EPO production and CKD anemia (17). In contrast with the profibrotic effect of myofibroblasts in CKD $(15,22$, 24), AKI myofibroblasts are activated transiently to enclose the injured tubular epithelium and promote tubular regeneration (26). Although further studies are needed to delineate the proreparative effect of AKI myofibroblasts, our findings emphasize the role of myofibroblasts in AKI-CKD transition due to the decrease of Acta 2 repressors by hypermethylation during AKI.

By mapping the fate of Col1a2-RFP ${ }^{+}$pericytes, we found that up to one-fifth of pericytes activated by AKI escaped apoptosis and became inactivated during recovery. In addition, our results showed that cell numbers of iPericytes did not return to the baseline level before AKI. Moreover, the cell numbers of iPericytes increased again with progressive renal fibrosis and function decline during follow-up. These findings suggested iPericytes
Figure 8. YBX2 expression in pericytes decreased after acute kidney injury. (A) Representative images showing the immunohistochemistry staining of YBX2 in the Ctrl kidneys. Arrows indicate YBX2+ interstitial cells. Scale bars: $25 \mu \mathrm{m}$. Original magnification, $\times 400$. (B) Representative images showing Col1a1-GFP+ $\mathrm{qPericytes}$ with YBX2 expression in the Ctrl kidney of Col1a1-GFP Tg mice. Scale bars: $25 \mu \mathrm{m}$. Original magnification, $\times 400$. (C) Representative images showing the immunohistochemistry staining of YBX2 in kidneys on day 7 after IRI-AKI. Arrowheads indicate $\mathrm{YBX} 2^{+}$tubular epithelial cells while asterisks indicate interstitial cells without YBX2. Scale bars: $25 \mu \mathrm{m}$. Original magnification, $\times 400$. (D) Dot chart showing the expression of $Y b \times 2$ in the $C$ trl and day 7 IRI-AKI kidneys. $n=5$. (E and F) Dot chart showing the expression of $Y b \times 2$ and Acta 2 in purified pericytes from the kidneys at the indicated time points after IRI-AKI. Horizontal bars represent the mean, error bars represent the SEM. $n=4 .{ }^{*} P<0.05$, ${ }^{* *} P<0.01$, ${ }^{* * *} P<$ 0.001 by 1 -way ANOVA with post hoc Tukey's correction. show a phenotype distinct from qPericytes. Mechanistic studies demonstrated that iPericytes retained a higher potential for cell proliferation, ECM production, and reactivation to aPericytes in response to injuries again.

We discovered the distinct populations of qPericytes, aPericytes (myofibroblasts), and iPericytes in kidneys before (normal control), on day 7 (AKI), and on day 28 (AKD) after AKI using a whole-mouse genome microarray. Because the potential of iPericytes for cell proliferation and ECM production was higher than that of qPericytes, and the expression of Acta2 in iPericytes was lower than that in aPericytes, our study further focused on the mechanisms underlying the change in activation status and function of these distinct pericyte populations. The epigenetic modifications in CKD myofibroblasts have been well studied, such as the hypermethylation of Rasal1, Epo, and Hif2a $(8,17,34)$. Burgeoning studies have also shown that epigenetic processes are involved in the pathogenesis of AKI (35). Because $\alpha \mathrm{SMA}$ is a robust activation marker for aPericytes (18), we studied the epigenetic regulation of Acta2, the expression of which is known to be transcriptionally upregulated by TGF- $\beta 1(7,36)$. Here, we identified that genes encoding Acta2 repressors, including Ybx2, Ybx1, Tead1, and Pura, were hypermethylated in aPericytes. Prolonged exposure to TGF-1 induced hypermethylation of the $Y b x 2$ promoter, thereby decreasing $Y b x 2$ expression. In aPericytes after treatment with demethylating agent 5-Aza, the expression of Ybx2, Timp3, Angpt1, and Angpt2 increased, the expression of Acta2 decreased, and the function for microvascular stabilization recovered.

In addition to the aforementioned evidence in pericyte cultures, we further demonstrated that 5-Aza treatment during AKI at a clinically relevant and nontoxic dose could reduce not only the accumulation of $\alpha \mathrm{SMA}^{+}$myofibroblasts and renal fibrosis but also the elevation of BUN and creatinine in mice on day 180 after AKI, showing the effect of demethylation on the prevention of AKI-CKD transition. In mice treated with 5-Aza after the first AKI, the proliferation and cell numbers of $\alpha \mathrm{SMA}^{+}$aPericytes and renal fibrosis in 
A - Short-term TGF- $\beta 1$ stimulation $(24 \mathrm{~h})$
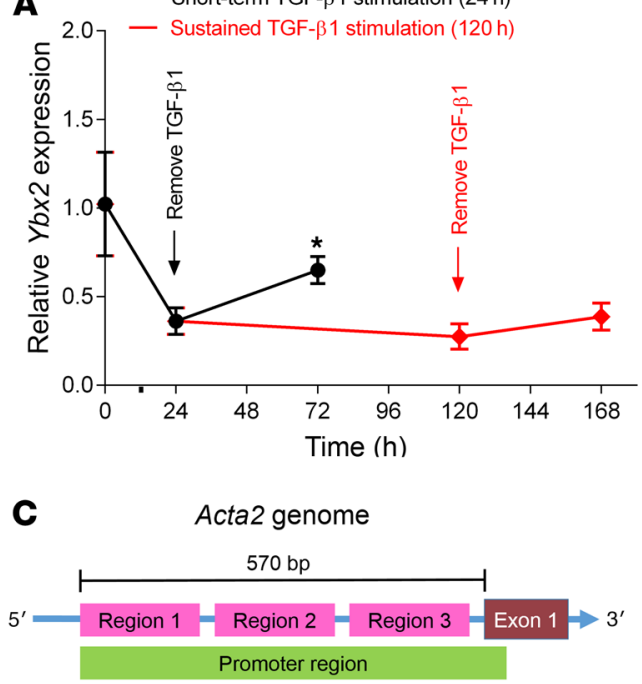

E

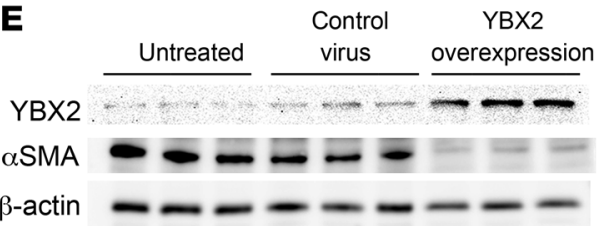

B

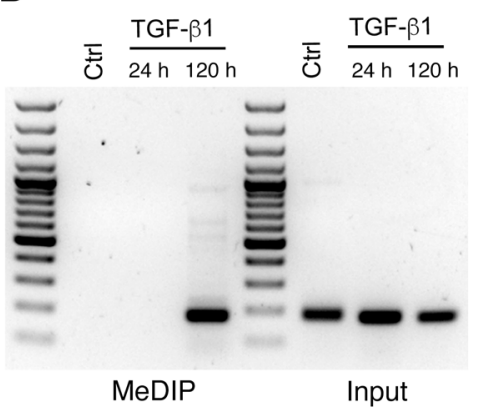

D
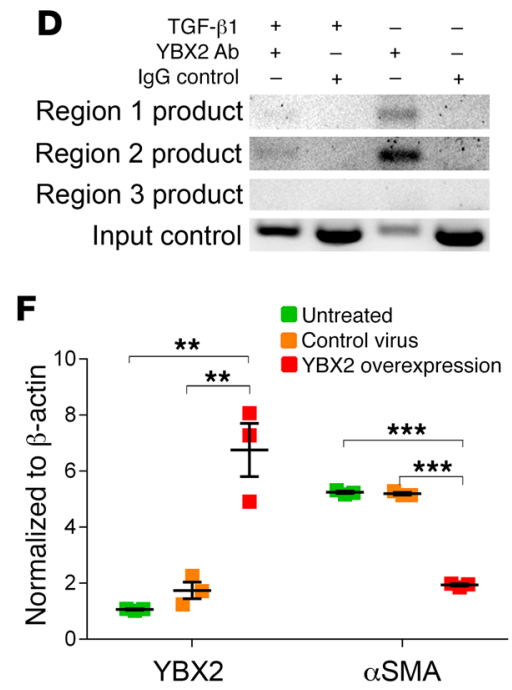

Figure 9. YBX2 repressed the expression of Acta2. (A) Line chart showing the expression of $Y b \times 2$ mRNA in the primary kidney pericytes after TCF- $\beta 1$ exposure and withdrawal at the indicated time points. Data were expressed as the mean \pm SEM. $n=3 .{ }^{*} P<0.05$ by $t$ test vs. before TGF- $\beta 1$ withdrawal. (B) Representative images showing the electrophoresis of the PCR products of the $Y b x 2$ gene using MeDIP or input DNA from primary kidney pericytes after TCF- $\beta 1$ exposure for 24 or 120 hours. Pericytes without TCF- $\beta 1$ exposure served as the Ctrl. (C) Scheme showing the primer design in the promoter regions of the Acta2 gene. (D) Representative images showing the electrophoresis of PCR products of the promoter of the Acta2 gene using DNA immunoprecipitated by antiYBX2 antibody (ChIP) or input DNA from the primary kidney pericytes with or without TCF- $\beta 1$ exposure for 120 hours. ChIP using isotype IgC served as the Ctrl. (E) Representative Western blot analyses for YBX2, $\alpha$ SMA, and $\beta$-actin in pericytes with or without lentiviral transduction for YBX2 expression. (F) Dot chart showing the relative expression of $Y B X 2$ and $\alpha S M A$ in pericytes with or without lentiviral transduction for YBX2 expression. Horizontal bars represent the mean, error bars represent the SEM. $n=3$. ${ }^{* *} P<0.01,{ }^{* *} P<0.001$ by 1 -way ANOVA with post hoc Tukey's correction. response to the second AKI were attenuated, showing the impact of methylation-induced epigenetic modification during the first AKI on cell proliferation and ECM production of iPericytes induced by a second AKI. All of these findings highlight that demethylation reduces aPericyte activation induced by AKI as well as reverses aPericytes to the phenotype of qPericytes, thereby preventing AKICKD transition and reducing the impact of repeated AKI on AKD or subclinical CKD. It was noteworthy that 5-Aza treatment did not provide a discernible effect on the recovery of renal function on day 28 after IRI-AKI, suggesting that, during AKI, the impact of demethylation on the repair of the injured tubular epithelium was negligible. Although a previous report showed that selective tubular cell ablation causes transient activation and migration of pericytes to enclose the injured tubular epithelium and promote tubular regeneration (26), 5-Aza treatment seemed not to change this function. Moreover, the finding of similar renal function impairment induced by a second AKI in mice with or without 5-Aza treatment emphasizes the negligible remote effect of demethylation on the response of kidneys to the second AKI. However, 5-Aza treatment during AKI induces long-term protective effects against renal fibrosis and function impairment as observed on day 180 after AKI.

The dynamic interplay between the repressors and activators collaborates in the regulation of Acta2 gene expression in myofibroblasts (31). YBX2, known as a member of the Y-box family of DNA/RNA-binding proteins, functions as a transcriptional regulator in the nucleus (37). It can bind to a Y-box element in the promoters of target genes and can also help stabilize mRNAs and regulate translation. YBX2 is highly expressed in germ cells and its deletion leads to infertility $(38,39)$. YBX2 expression and function in the kidney have not been shown before. Our data showed that $Y b x 2$ expression was decreased in aPericytes and recovered partially in iPericytes, a pattern opposite of the change of Acta2 expression. Mechanistically, we confirmed that YBX2 protein bound to the promoter region of Acta2 and YBX2 overexpression decreased $\alpha$ SMA expression, demonstrating YBX2 as a repressor of $\alpha$ SMA. Previous evidence has shown that the Acta2 promoter is bound by PUR $\alpha, \mathrm{YBX} 1$, and TEAD1 repressors in quiescent fibroblasts $(29,31,40)$. In the presence of TGF- $\beta 1$, conformational changes occur and repressor proteins are removed from the promoter. TGF- $\beta 1$ exposure for 1 day transiently decreased $Y b x 2$ mRNA in qPericytes. However, 5-day TGF- $\beta 1$ exposure resulted in the methylation of the $Y b x 2$ promoter, resulting in prolonged repression of $Y b x 2$ mRNA even after TGF- $\beta 1$ was withdrawn. In addition to $Y b x 2$, we also showed methylation in the promoters of Tead1, Pura, and Ybx1 in aPericytes. Through the methylation of Acta2 repressor genes during AKI, iPericytes retained the epigenetic modification with a higher potential for reactivation by repeated injuries and may promote the transition toward CKD.

Why the expression of YBX2 increased in injured tubular epithelial cells after AKI is not clear and needs further study. Injured tubular epithelial cells may dedifferentiate after AKI, but they do not express $\alpha \mathrm{SMA}$ and migrate through the base- 
ment membrane to become myofibroblasts $(22,41)$. Increased Acta2 repressors including YBX2 might be one mechanism to maintain cells in the epithelial phenotype.

In conclusion, AKI activates qPericytes to aPericytes, namely myofibroblasts, of which one-fifth escape apoptosis and become nonquiescent iPericytes during renal recovery. These iPericytes retain methylation-induced repression of Acta2 repressors including $Y b x 2$ and show a higher potential to promote AKI-CKD transition or fibrogenesis upon repeated AKI. Demethylation by 5-Aza during AKI/AKD may provide a strategy to prevent CKD development.

\section{Methods}

Animals. Col1a1-GFP ${ }^{T g}$ transgenic reporter mice were generated in the C57BL/6 background as previously described (15). Col1a2-CreERT $T^{T_{g}}$ transgenic mice were generated using a 6-kb Col1a2 enhancer to induce the expression of a cDNA encoding the CreERT fusion protein

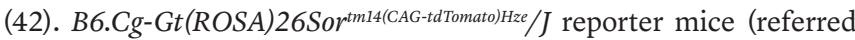
to herein as ROSA2 $6^{\text {fstdomato/+) }}$, B6 129S4-Foxd $1^{\text {tml(GFP/(re)Amc } / J ~(r e f e r r e d ~}$ to herein as Foxd $1^{\mathrm{Cre} /+}$ ), and $\mathrm{C} 57 \mathrm{BL} / 6 \mathrm{WT}$ mice were obtained from The Jackson Laboratory.

Mouse models of acute kidney injury. Adult (8-10 weeks) male mice were anesthetized with ketamine/xylazine $(100 / 10 \mathrm{mg} / \mathrm{kg}$, intraperitoneally) and subjected to right Nx. After 2 weeks, the left kidney was clamped for 22 minutes with a nontraumatic microaneurysm clip to induce IRI under the homeothermic blanket system (Stoelting Co.) to maintain the core body temperature at $37^{\circ} \mathrm{C}$. Only the right $\mathrm{Nx}$ was performed in the Ctrl mice. Acute adenine nephropathy was induced in adult mice that were fed a pellet diet of LabDiet 5001 (TestDiet) containing $0.25 \%$ adenine (MilliporeSigma) for 5 days (43). Mice on a regular pellet diet of LabDiet 5001 served as Ctrl.

Isolation and culture of kidney pericytes. Pericytes were purified from kidneys using a previously described method (17). Briefly, the kidney was decapsulated, diced, and then incubated at $37^{\circ} \mathrm{C}$ for 40 minutes with collagenase $(50 \mathrm{mg} / \mu \mathrm{L}$, Roche Applied Science) and dispase (0.3 U/ $\mu \mathrm{L}$, Roche Applied Science) in Hanks' Balanced Salt Solution (MilliporeSigma). After centrifugation, cells were resuspended in $3 \mathrm{~mL} \mathrm{PBS} / 1 \%$ BSA and filtered $(40 \mu \mathrm{m})$. For the transcriptome analysis, pericytes were isolated from the single-cell suspension by sorting $\mathrm{GFP}^{+} \mathrm{RFP}^{+} \mathrm{F} 4 / 80-\mathrm{APC}^{-}$ CD31-APC-CD324-APC- cells from Foxd1 ${ }^{\text {Cre/+ }}$ ROSA26 $6^{\text {stdTomato/+ Colla1- }}$ $G F P^{T g}$ mice using a FACSAria cell sorter (BD Biosciences). Microarray data have been submitted to the MIAME compliant Gene Expression Omnibus (GEO) database (accession number GSE140988). In the rest of the experiments, pericytes were obtained by sorting $\mathrm{GFP}^{+}$cells from Col1a1-GFP ${ }^{T g}$ mice and cultured in DMEM with $10 \%$ FBS. Passage 0 cells were used for experiments. RNA and genomic DNA were extracted for experiments. In TGF- $\beta 1$ exposure experiments, cells were washed with $1 \times$ PBS and supplied with culture medium with or without $5 \mathrm{ng} / \mathrm{mL}$ TGF- $\beta 1$ (R\&D Systems) (Figure 3 and Figure 9). In aPericytes culture, cells were treated with $500 \mathrm{ng} / \mathrm{mL} 5$-Aza (MilliporeSigma) or vehicle for 3 days, followed by TGF- $\beta 1$ treatment for 16 hours (Supplemental Figure 11). Cellular RNA was then extracted for experiments.

Cell proliferation assay. Cell proliferation was evaluated using a 3-(4,5-Dimethylthiazol-2-yl)-2,5-diphenyltetrazolium bromide (MTT, MilliporeSigma) assay. Pericytes were seeded into a 96-well microplate at a density of $1 \times 10^{4}$ cells/well. The kinetics of cell proliferation in pericytes was detected on days $0,3,5$, and 7 . The optical density (OD) at the wavelength of $490 \mathrm{~nm}$ was used to evaluate cell proliferation.
Administration of 5-azacytidine to the mouse models of acute kidney injury. Mice received 2 weeks of treatment by daily subcutaneous injections of either PBS vehicle or $5-\mathrm{Aza}(0.5 \mathrm{mg} / \mathrm{kg}$, MilliporeSigma) for 5 days per week after IRI as outlined in Figure 5A and Figure 6A.

Three-dimensional vascular tube regression assay. Isolated kidney pericytes from Col1a1-GFP ${ }^{T g}$ mice and HUVECs (ScienCell Research Laboratories) were cocultured at a ratio of 1:8 in $3 \mathrm{D}$ vascular tube regression assays following a modified protocol as previously described (9). HUVECs $\left(6 \times 10^{5}\right.$ cells $\left./ \mathrm{mL}\right)$ were suspended in $3 \mathrm{mg} / \mathrm{mL}$ rat tail collagen type I at $4^{\circ} \mathrm{C}$ (Invitrogen), transferred to a $37^{\circ} \mathrm{C}$ incubator, and were allowed to form 3D networks in the presence of vascular endothelial growth factor $165(40 \mathrm{ng} / \mathrm{mL})$ and fibroblast growth factor 2 (40 ng/mL) (PeproTech) in 384-well microplates (ibidi Inc.). Twentyfour hours later, human plasma kallikrein (KLK) (Enzyme Research Laboratories) was added to the cultures. Cultures were monitored for gel contraction and the gel-regressed area and vessel density were quantified at indicated time points by Image $1.42(\mathrm{NIH})$.

Magnetic methylated DNA immunoprecipitation (MeDIP). Genomic DNA was extracted from cultured primary pericytes using the DNeasy Blood \& Tissue Kit (QIAGEN). DNA was then sheared by sonication (Bioruptor Pico, Biosearch Technologies) to generate fragments between 100-600 bp. Using the MagMeDIP kit (Diagenode), the Magbead-bound 5-methylcytosine antibody was used to immunoprecipitate methylated DNA. The beads were captured by a magnet to remove the supernatant. After washing, we resuspended the beads and eluted the immunoprecipitated DNA for PCR to detect the target DNA sequence.

Chromatin immunoprecipitation (ChIP). Using a ChIP kit (Magna ChIP A/G, MilliporeSigma), we cross-linked the protein-DNA complexes by incubating pericytes with $1 \%$ formaldehyde. The reaction was stopped by adding glycine. The pericytes were harvested and sonicated to shear the chromatin to an average length of about $1 \mathrm{~kb}$. We then centrifuged the lysates for 10 minutes and collected the supernatant. Both anti-YBX2 antibody (13538-1-AP, Proteintech) and magnetic beads were added in the supernatant and incubated overnight at $4^{\circ} \mathrm{C}$. Then, the sample was loaded on a magnetic separator and the supernatant was removed completely. The magnetic bead-antibody/ chromatin complex was washed by resuspending the beads in cold buffers. We eluted and purified the DNA for PCR to detect the presence of the Ybx2 DNA sequence.

Lentiviral transduction. For the overexpression of $\mathrm{YBX} 2, \mathrm{mYbx} 2$ (OriGene Technologies) was cloned into a Lentivector pCDH-CMVEF1-GFP+Puro. Lentivirus was generated by RNA Technology Platform and Gene Manipulation Core, Academia Sinica. GFP virus (pAS7w.EGFP.puro) was used as the control. Pericytes were transduced by the virus to overexpress YBX2.

Bisulfite genomic sequencing (BGS). Genomic DNA was prepared from pericytes. Sodium bisulfite conversion of genomic DNA was performed using the EZ DNA Methylation Kit (ZYMO Research). The bisulfite-converted genomic DNA was used for PCR to amplify the promoter of $Y b x 2$ using the primers shown in Supplemental Table 2. The PCR products were then gel-purified with the QIAquick Gel Extraction Kit (QIAGEN). The eluted DNA fragments were ligated into the pGEM-T Easy Vector (Promega Corporation) and 4 colonies for each mouse were randomly chosen for sequencing.

Temporal induction of Cre recombinase activity by tamoxifen administration. Tamoxifen in olive oil $(10 \mathrm{mg} / \mathrm{mL}$ ) (MilliporeSigma) was prepared by sonication and was administered intraperitoneally into 
Col1a2-CreERT ${ }^{T g}$ mice at $1 \mathrm{mg}$ daily for 5 days at the age of 8 to 9 weeks (44). After a 10-day washout period, mice were subjected to AKI.

Tissue preparation and histology. For immunofluorescence staining, mouse tissues were prepared and stained as previously described (17). Primary antibodies against the following proteins were used for immunolabeling in $5 \mu$ m-thick cryosections: $\alpha$ SMA-Cy3 (C6198, clone 1A4), aSMA-FITC (F3777, clone 1A4), laminin (MilliporeSigma), DNMT1 (5032, Cell Signaling Technology), cleaved caspase-3 (9664, Cell Signaling Technology), CD31 (14-0311-82, Invitrogen), F4/80 (14-4801-82, Invitrogen), YBX2 (ab33164, Abcam), and Ki67 (Ab15580, Abcam). Nonimmunized rabbit IgG (011-000-003, Jackson ImmunoResearch Laboratories) was used as a control for YBX2 staining. Apoptosis staining was performed by TUNEL assay (G3250, Promega Corporation). Fluorescence-conjugated secondary antibody labeling (Jackson ImmunoResearch Laboratories), 4',6-diamidino-2-phenylindole (DAPI) staining, Vectashield mounting (Vector Laboratories), and image capture and processing were carried out as previously described (44). Conventional and confocal images were taken with a Zeiss Axio Imager A1 Microscope with AxioVision Software and a Zeiss Laser Scanning 880 Microscope with Zen 2011 Software, respectively (Carl Zeiss). Quantification of specific cells in tissue sections was carried out as follows. In brief, the $\mathrm{GFP}^{+}, \mathrm{RFP}^{+}$, and $\mathrm{DAPI}^{+}$cells were identified by green, red, and blue fluorescence, respectively. The $\alpha \mathrm{SMA}^{+}$cells were identified by the presence of Cy3 or FITC fluorescence in greater than $75 \%$ of the cell area immediately surrounding the nuclei detected by DAPI. Specific cells were counted in 10 randomly selected cortical interstitial fields per mouse at $\times 400$ magnification (high-powered field). For immunohistochemistry staining, kidneys were fixed in formalin and embedded in paraffin. After deparaffinization, antigen retrieval was performed by heating in 10 $\mathrm{mM}$ sodium citrate buffer ( $\mathrm{pH}$ 6.0) for 15 minutes and subsequently incubating for 15 minutes in 3\% hydrogen peroxide to block endogenous peroxidase. Sections were blocked in $10 \%$ normal goat serum for 1 hour, and then incubated with the primary antibodies against YBX2 (13538-1-AP, Proteintech) or nonimmunized rabbit IgG (011000-003, Jackson ImmunoResearch Laboratories) at $4^{\circ} \mathrm{C}$ overnight. A polyclonal horseradish peroxidase-conjugated goat anti-rabbit antibody (GTX213110-01, GeneTex) was used to bind the primary antibody. 3-3'-Diaminobenzidine (RE7230-K, Novolink) was used in the detection procedure. Interstitial fibrosis was quantified in the Picrosirius red-stained paraffin sections.

Polymerase chain reaction (PCR). Total RNA was extracted using the RNeasy Mini Kit (QIAGEN). cDNA was synthesized using the iScript cDNA Synthesis Kit (Bio-Rad). PCR or quantitative PCR was performed using methods previously described (17). The expression levels were normalized to glyceraldehyde 3-phosphate dehydrogenase gene (Gapdh). The specific primer pairs used in quantitative PCR and PCR are listed in Supplemental Table 1 and Supplemental Table 2, respectively.

Western blot analysis. Total cellular protein extracted using the radioimmunoprecipitation assay buffer was subjected to Western blot analysis using previously described methods (45). The following primary antibodies were used to detect the specific protein: YBX2 (13538-
1-AP, Proteintech), aSMA (ab125057, Abcam), DNMT1 (5032, Cell Signaling Technology), and $\beta$-actin (4967, Cell Signaling Technology).

Statistics. Data were expressed as the mean \pm standard error of the mean (SEM). One-way ANOVA with post hoc Tukey's correction was used for the comparison between each group. One-way ANOVA with post hoc Dunnett's correction was used for the comparison between the control group and each of the other groups. Two-tailed Student's $t$ test was used to compare 2 different groups. A $P$ value less than 0.05 was considered significant. Statistical analyses were carried out using the GraphPad Prism software.

Study approval. All of the animal experiments were executed under a protocol approved by the Institutional Animal Care and Use Committee of the National Taiwan University College of Medicine.

\section{Author contributions}

YHC, SYP, YHS, HMS, SYW, CFL, and WCC carried out experiments and analyzed data. YHC, CS, KCY, LCL, YMC, and TSC participated in experiment design and data analysis. SLL designed and directed the project. YHC, TSC, and SLL wrote the manuscript.

\section{Acknowledgments}

The authors thank Shu-Yi Huang (National Taiwan University Hospital [NTUH]) for helpful discussion and editing the manuscript, David Brenner (UCSD, San Diego, CA) for Col1a1-GFP ${ }^{T g}$ mice, Christopher P. Denton (University College London, London, United Kingdom) and Andrew Leask (University of Western Ontario, Ontario, Canada) for Col1a2-CreERT ${ }^{T g}$ mice, Ya-Chun Tu (NTUH) for technical assistance, the Department of Medical Research of NTUH for equipment support, the Cell Sorting Core Facility of the First Core Laboratory, the Transgenic Mouse Model Core Facility of the National Core Facility Program for Biotechnology, Ministry of Science and Technology (MOST), the Gene Knockout Mouse Core Laboratory of the National Taiwan University (NTU) Center of Genomic Medicine, and the RNA Technology Platform and Gene Manipulation Core (Academia Sinica, Taipei, Taiwan). YHC was supported by MOST (104-2314-B-002-119-MY3, 1072314-B-002-020, 108-2314-B-002-060), NTUH (107-003974), NTUH Jin-Shan Branch (108-01), and the Mrs. Hsiu-Chin Lee Kidney Research Foundation. SYP was supported by MOST (1062314-B-418-006, 107-2314-B-418-001). YMC was supported by MOST (108-2314-B-002-064-MY3). SLL was supported by MOST (107-2314-B-002-078, 108-2314-B-002-078-MY3, 108-2314-B002-012), National Health Research Institutes (EX108-10633SI), NTUH (107-T02), and Taiwan Health Foundation.

Address correspondence to: Shuei-Liong Lin, Graduate Institute of Physiology, Number 1, Jen-Ai Road Section 1, Taipei, Taiwan 100. Phone: 886.2.23123456 ext.88235; Email: linsl@ntu.edu. tw. Or to: Tzong-Shinn Chu, Renal Division, Department of Medicine, National Taiwan University Hospital, Number 7, ChungShan South Road, Taipei, Taiwan 100. Phone: 886.2.23123456 ext.65403; Email: tschu@ntu.edu.tw.
1. Chawla LS, Eggers PW, Star RA, Kimmel PL. Acute kidney injury and chronic kidney disease as interconnected syndromes. $\mathrm{NEnglJ} \mathrm{Med}$. 2014;371(1):58-66.
2. Lai CF, et al. Kidney function decline after a non-dialysis-requiring acute kidney injury is associated with higher long-term mortality in critically ill survivors. Crit Care. 2012;16(4):R123.
3. Kellum J, et al. Kidney disease: Improving global outcomes (KDIGO) acute kidney injury work group. KDIGO clinical practice guideline for acute kidney injury. Kidney Int Suppl. 2012;2(1):1-138. 
4. Cheng SY, et al. Losartan reduces ensuing chronic kidney disease and mortality after acute kidney injury. Sci Rep. 2016;6:34265.

5. Ferenbach DA, Bonventre JV. Mechanisms of maladaptive repair after AKI leading to accelerated kidney ageing and CKD. Nat Rev Nephrol. 2015;11(5):264-276.

6. Yang L, Besschetnova TY, Brooks CR, Shah JV, Bonventre JV. Epithelial cell cycle arrest in G2/M mediates kidney fibrosis after injury. Nat Med. 2010;16(5):535-543.

7. Wu CF, et al. Transforming growth factor $\beta-1$ stimulates profibrotic epithelial signaling to activate pericyte-myofibroblast transition in obstructive kidney fibrosis. Am J Pathol. 2013;182(1):118-131.

8. Bechtel W, et al. Methylation determines fibroblast activation and fibrogenesis in the kidney. Nat Med. 2010;16(5):544-550.

9. Schrimpf C, et al. Pericyte TIMP3 and ADAMTS1 modulate vascular stability after kidney injury. JAm Soc Nephrol. 2012;23(5):868-883.

10. Lin SL, et al. Targeting endothelium-pericyte cross talk by inhibiting VEGF receptor signaling attenuates kidney microvascular rarefaction and fibrosis. Am J Pathol. 2011;178(2):911-923.

11. Sato $Y$, et al. Heterogeneous fibroblasts underlie age-dependent tertiary lymphoid tissues in the kidney. JCI Insight. 2016;1(11):e87680.

12. Saito H, et al. Persistent expression of neutrophil gelatinase-associated lipocalin and M2 macrophage markers and chronic fibrosis after acute kidney injury. Physiol Rep. 2018;6(10):e13707.

13. Chou YH, et al. Renin-angiotensin system inhibitor is associated with lower risk of ensuing chronic kidney disease after functional recovery from acute kidney injury. Sci Rep. 2017;7:46518.

14. Chou YH, Huang TM, Chu TS. Novel insights into acute kidney injury-chronic kidney disease continuum and the role of renin-angiotensin system. J Formos Med Assoc. 2017;116(9):652-659.

15. Lin SL, Kisseleva T, Brenner DA, Duffield JS. Pericytes and perivascular fibroblasts are the primary source of collagen-producing cells in obstructive fibrosis of the kidney. Am J Pathol. 2008;173(6):1617-1627.

16. Chen YT, et al. Platelet-derived growth factor receptor signaling activates pericyte-myofibroblast transition in obstructive and post-ischemic kidney fibrosis. Kidney Int. 2011;80(11):1170-1181.

17. Chang YT, et al. DNA methyltransferase inhibition restores erythropoietin production in fibrotic murine kidneys. J Clin Invest. 2016;126(2):721-731.

18. Lemos DR, et al. Maintenance of vascular integrity by pericytes is essential for normal kidney function. Am J Physiol Renal Physiol. 2016;311(6):F1230-F1242.

19. Kramann R, Wongboonsin J, Chang-Panesso M,
Machado FG, Humphreys BD. Gli1 ${ }^{+}$pericyte loss induces capillary rarefaction and proximal tubular injury. J Am Soc Nephrol. 2017;28(3):776-784.

20. Shih HM, Wu CJ, Lin SL. Physiology and pathophysiology of renal erythropoietin-producing cells. J Formos Med Assoc. 2018;117(11):955-963.

21. Stratman AN, Schwindt AE, Malotte KM, Davis GE. Endothelial-derived PDGF-BB and HB-EGF coordinately regulate pericyte recruitment during vasculogenic tube assembly and stabilization. Blood. 2010;116(22):4720-4730.

22. Humphreys BD, et al. Fate tracing reveals the pericyte and not epithelial origin of myofibroblasts in kidney fibrosis. Am J Pathol. 2010;176(1):85-97.

23. Chang FC, Chou YH, Chen YT, Lin SL. Novel insights into pericyte-myofibroblast transition and therapeutic targets in renal fibrosis. J Formos Med Assoc. 2012;111(11):589-598.

24. Kramann R, et al. Perivascular Gli1+ progenitors are key contributors to injury-induced organ fibrosis. Cell Stem Cell. 2015;16(1):51-66.

25. Souma T, et al. Erythropoietin synthesis in renal myofibroblasts is restored by activation of hypoxia signaling. J Am Soc Nephrol. 2016;27(2):428-438.

26. Schramek H, et al. Neuropilin-1 and neuropilin-2 are differentially expressed in human proteinuric nephropathies and cytokine-stimulated proximal tubular cells. Lab Invest. 2009;89(11):1304-1316.

27. Lin SL, et al. Macrophage Wnt7b is critical for kidney repair and regeneration. Proc Natl Acad Sci USA. 2010;107(9):4194-4199.

28. Endo T, et al. Exploring the origin and limitations of kidney regeneration. J Pathol. 2015;236(2):251-263.

29. Liu F, Wang X, Hu G, Wang Y, Zhou J. The transcription factor TEAD1 represses smooth muscle-specific gene expression by abolishing myocardin function. J Biol Chem. 2014;289(6):3308-3316.

30. Subramanian SV, Polikandriotis JA, Kelm RJ, David JJ, Orosz CG, Strauch AR. Induction of vascular smooth muscle alpha-actin gene transcription in transforming growth factor beta1-activated myofibroblasts mediated by dynamic interplay between the Pur repressor proteins and Sp1/Smad coactivators. Mol Biol Cell. 2004;15(10):4532-4543.

31. Strauch AR, Hariharan S. Dynamic interplay of smooth muscle $\alpha$-actin gene-regulatory proteins reflects the biological complexity of myofibroblast differentiation. Biology (Basel). 2013;2(2):555-586.

32. Zhang A, et al. YB-1 coordinates vascular smooth muscle alpha-actin gene activation by transforming growth factor beta 1 and thrombin during differentiation of human pulmonary myofibro- blasts. Mol Biol Cell. 2005;16(10):4931-4940.

33. Rodemann HP, Müller GA. Characterization of human renal fibroblasts in health and disease: II. In vitro growth, differentiation, and collagen synthesis of fibroblasts from kidneys with interstitial fibrosis. Am J Kidney Dis. 1991;17(6):684-686.

34. Sato K, et al. An immortalized cell line derived from renal erythropoietin-producing (REP) cells demonstrates their potential to transform into myofibroblasts. Sci Rep. 2019;9(1):11254.

35. Tang J, Zhuang S. Epigenetics in acute kidney injury. Curr Opin Nephrol Hypertens. 2015;24(4):351-358.

36. Rahaman SO, et al. TRPV4 mediates myofibroblast differentiation and pulmonary fibrosis in mice. JClin Invest. 2014;124(12):5225-5238.

37. Yu J, Hecht NB, Schultz RM. Requirement for RNA-binding activity of MSY2 for cytoplasmic localization and retention in mouse oocytes. Dev Biol. 2003;255(2):249-262.

38. Yang J, et al. Absence of the DNA-/RNA-binding protein MSY2 results in male and female infertility. Proc Natl Acad Sci USA. 2005;102(16):5755-5760.

39. Yan C, et al. CFTR deletion in mouse testis induces VDAC1 mediated inflammatory pathway critical for spermatogenesis. PLoS ONE. 2016;11(8):e0158994.

40. Knapp AM, Ramsey JE, Wang SX, Godburn KE, Strauch AR, Kelm RJ. Nucleoprotein interactions governing cell type-dependent repression of the mouse smooth muscle alpha-actin promoter by single-stranded DNA-binding proteins Pur alpha and Pur beta. J Biol Chem. 2006;281(12):7907-7918.

41. Kusaba T, Lalli M, Kramann R, Kobayashi A, Humphreys BD. Differentiated kidney epithelial cells repair injured proximal tubule. Proc Natl Acad Sci USA. 2014;111(4):1527-1532.

42. Zheng B, Zhang Z, Black CM, de Crombrugghe B, Denton CP. Ligand-dependent genetic recombination in fibroblasts: a potentially powerful technique for investigating gene function in fibrosis. Am J Pathol. 2002;160(5):1609-1617.

43. Tamura M, Aizawa R, Hori M, Ozaki H. Progressive renal dysfunction and macrophage infiltration in interstitial fibrosis in an adenine-induced tubulointerstitial nephritis mouse model. Histochem Cell Biol. 2009;131(4):483-490.

44. Chen YT, et al. Lineage tracing reveals distinctive fates for mesothelial cells and submesothelial fibroblasts during peritoneal injury. J Am Soc Nephrol. 2014;25(12):2847-2858.

45. Lin SL, et al. Pentoxifylline attenuates tubulointerstitial fibrosis by blocking Smad3/4-activated transcription and profibrogenic effects of connective tissue growth factor. J Am Soc Nephrol. 2005;16(9):2702-2713. 